\title{
Recent findings and future directions for interpolar mitotic kinesin inhibitors in cancer therapy
}

\begin{abstract}
The kinesin class of microtubule-associated motor proteins present attractive anticancer targets owing to their roles in key functions in dividing cells. Two interpolar mitotic kinesins Eg5 and HSET have opposing motor functions in mitotic spindle assembly with respect to microtubule movement, but both offer opportunities to develop cancer selective therapeutic agents. Here, we summarize the progress to date in developing inhibitors of Eg5 and HSET, with an emphasis on structural biology insights into the binding modes of allosteric inhibitors, compound selectivity and mechanisms of action of different chemical scaffolds. We discuss translation of preclinical studies to clinical experience with Eg5 inhibitors, recent findings on potential resistance mechanisms and explore the implications for future anticancer drug development against these targets.
\end{abstract}

First draft submitted: 23 October 2015; Accepted for publication: 20 January 2016; Published online: 15 March 2016

Keywords: allosteric $\bullet$ Eg5 $\bullet$ HSET $\bullet$ inhibition $\bullet$ kinesin $\bullet$ mitosis $\bullet$ resistance

\section{Interpolar mitotic kinesins: function \& structure}

Chemotherapeutic agents which target microtubules have been used to treat various cancer types. Microtubules are formed from the polymerization of tubulin monomers, and microtubule-targeting drugs may hyperstabilize GDP-bound polymerized tubulin, for example, taxanes and epothilones, or act by destabilizing and depolymerizing microtubules, for example, vinca alkaloids and colchicine. Both classes of molecule hinder the dynamic processes of microtubule formation and remodeling. This is particularly important in cell division, where tubulin polymerization and depolymerization is essential for the formation of a normal mitotic spindle [1]. Although clinically successful, the significant neuropathic side effects associated with the use of microtubule-targeting agents that act as mitotic spindle poisons are dose limiting. Furthermore, resistance to these drugs is common $[2,3]$. Alternative strategies to spe- cifically target the mitotic spindle in cancer cells have been explored. The kinesin class of microtubule-associated motor proteins represent attractive anticancer targets owing to their roles in key functions in dividing cells.

The role of kinesins in mitosis

The kinesin superfamily consists of over 650 distinct proteins which are categorized into 14 subfamilies according to their structural similarity and function. Over 70 of these proteins belong to the kinesin-5 family and are found in eukaryotic organisms [4]. The mitotic kinesins (in collaboration with the dyneins, another important family of microtubule-associated motor proteins) are integral force generators in the process of cell division, ensuring that chromosomes are separated with the highest integrity [5]. Kinesins use ATP hydrolysis to generate movement along microtubules by means of conformational changes in the protein [6]. Their relatively straightforward yet varying individual
Stephanie M Myers*,1 \& Ian Collins**,1

${ }^{1}$ Cancer Research UK Cancer Therapeutics Unit, The Institute of Cancer Research, London SM2 5NG, UK *Author for correspondence: stephanie.myers@icr.ac.uk

**Author for correspondence: ian.collins@icr.ac.uk 
roles in spindle dynamics result collectively in complex behavior [7].

There are currently 16 kinesins implicated in coordinating aspects of mitosis and cytokinesis [8]. In this review, we focus on two mitotic kinesins which are located primarily on the interpolar microtubules. These structures extend from one spindle pole across the cell equator and interdigitate with opposing interpolar microtubules, exerting additional pull on the chromosomes thus stabilizing the mitotic spindle [9]. The most extensively studied of the mitotic kinesins is $\mathrm{Eg} 5$ (also known as KIF11, kinesin-5 or KSP) and is involved in formation of the bipolar spindle, which requires sustained outward forces for its maintenance [10]. $\mathrm{Eg} 5$ is thought to exist as a homotetramer which cross-links two opposing interpolar microtubules, pushing them apart as a result of its plus-end-directed motility [8]. The closely related plus-end-directed kinesin KIF15 has been shown to act cooperatively with $\mathrm{Eg} 5$ to promote bipolar spindle assembly [11,12]. In contrast, HSET (also known as KIFC1) is a minus-end-directed motor, acting antagonistically toward $\mathrm{Eg} 5$ but whose role is also in bipolar spindle assembly [13-15]. HSET has also been implicated in microtubule organization and promoting stability of the central spindle, a requirement for cytokinesis to be performed correctly (Figure 1) [16].

\section{The structure of kinesins}

Understanding the structure and function of a protein target to underpin the development of clinical candidates is pivotal in modern drug discovery. Throughout, we will make reference to the key structural features of the Eg5 and HSET kinesins, as illustrated in the Eg5ADP-Mg crystal structure (Figure 2), with particular emphasis on experimentally determined or proposed inhibitor-bound states.

All cytoskeletal motors, including kinesins, consist of a catalytic motor domain (head) which contains two binding domains, one for ATP and one for microtubules. Kinesins can be classified into N-type kinesins where the motor domain is at the $\mathrm{N}$-terminus (e.g., Eg5), M-type where the motor domain is flanked by other domains or C-type where the motor domain is close to the C-terminus (e.g., HSET) [17]. Kinesins typically have two globular motor domains, attached to a coiled-coil stalk (neck) region by neck linkers. At the opposite end of the stalk, is a tail region which is responsible for interacting with specific cargos and adaptor proteins [18].

The motor domains of the kinesin superfamily, consisting of ca. 350 amino acids, are highly conserved and thus Eg5 and HSET bear strong structural resemblance in these regions despite their opposing mitotic functions. $\mathrm{Eg} 5$ consists of eight antiparallel $\beta$ strands

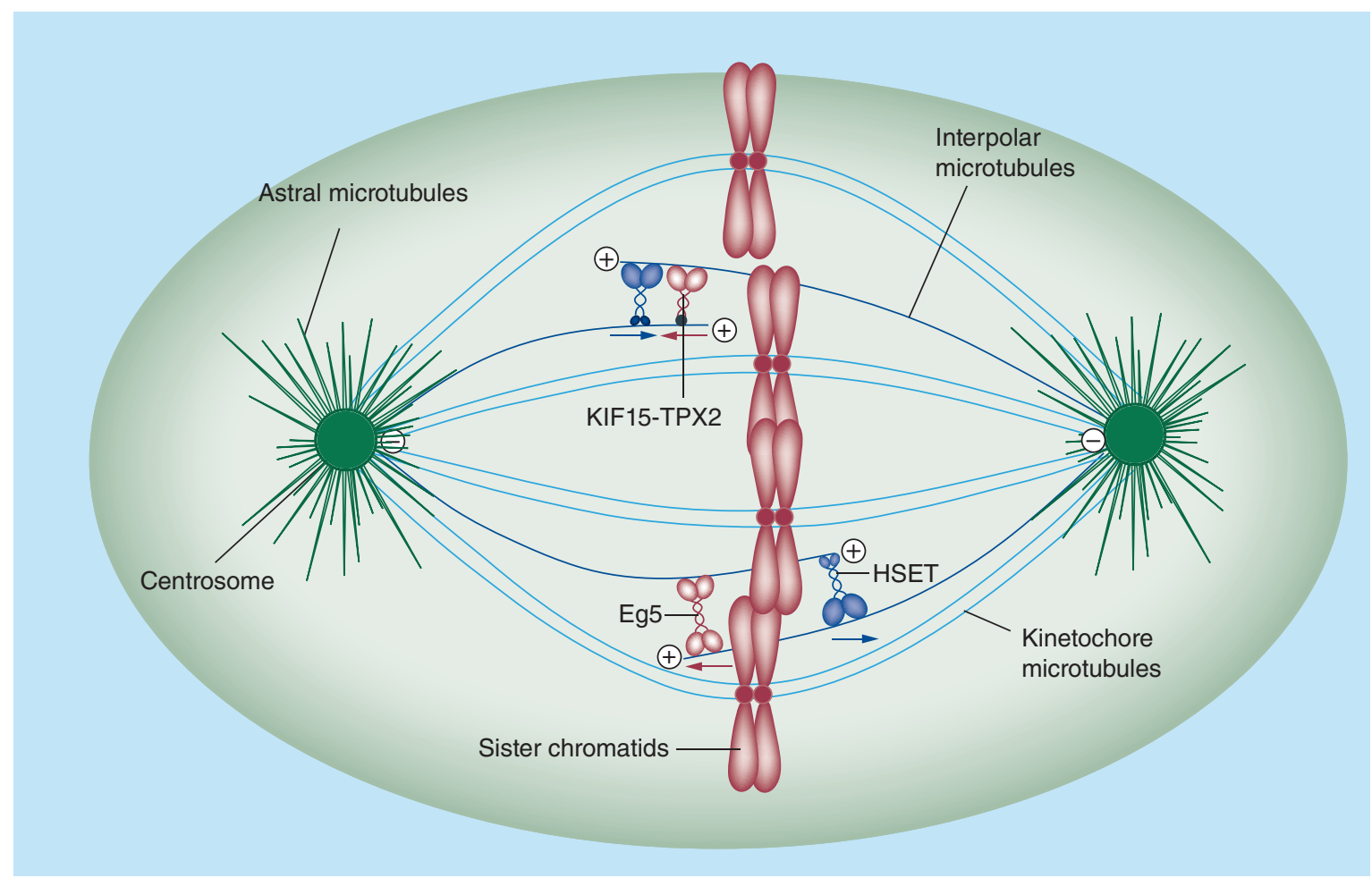

Figure 1. Simplified representation of the mitotic spindle architecture and relevant mitotic kinesins located on intrapolar microtubules.

Adapted with permission from [8] @ Macmillan Publishers (2012). 


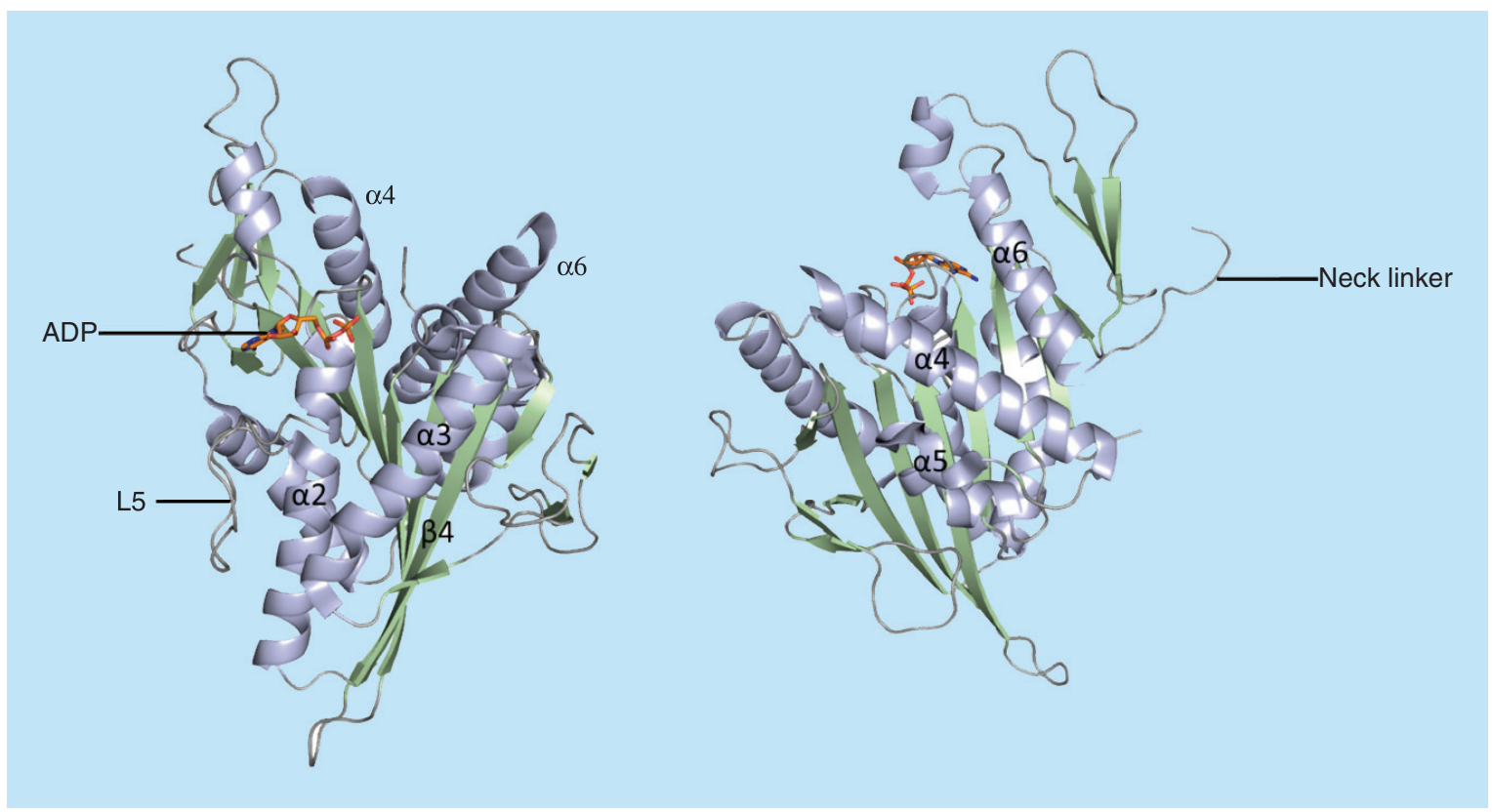

Figure 2. Crystal structure of Eg5-ADP-Mg (PDB 1II6); two alternative views. $\alpha$-helices are shown in light blue, $\beta$-sheets in pale green and loop regions in gray. ADP is shown in stick form (orange).

at its core, flanked by three major $\alpha$-helices on either side. The catalytic site is situated above the central $\beta$-sheet, surrounded by the phosphate-binding loop (P-loop; between $\alpha 2$ and $\beta 3$ ) which tightly binds the $\beta$-phosphate group of ATP/ADP, and switch motifs I (between $\alpha 3$ and $\beta 6$ ) and II (between $\alpha 4$ and $\beta 7$ ) which change their conformation depending on the presence or absence of a $\gamma$-phosphate group [17,19]. The $\alpha 2$ helix next to the P-loop is unusual; the helical repeat is intersected by the surface-exposed loop 5 that has been proposed to modulate $\mathrm{Eg} 5$ function by interacting with the catalytic site. The loop 5 motif is common to both HSET and Eg5, but the difference in amino acid chain length is striking (Figure 3). While the loop 5 element of $\mathrm{Eg} 5$ consists of 17 amino acids [20], it has been reported that kinesin-14 family members can have as few as three [21].

At the C-terminus of the motor domain, the neck linker connects to the stalk domain and adopts an immobilized 'docked' conformation when the motor domain is bound to microtubules. However, in the absence of microtubules, it reverts to a more mobile 'undocked' conformation. The neck linker enables $\mathrm{Eg} 5$ movement in the direction of the plus end of the microtubule by extending toward it, driving motility in this direction [22]. Notably, in the HSET-ADP-Mg crystal structure, the neck linker is unresolved, suggesting a high degree of flexibility in its nucleotide-bound state. However, it has been suggested that the ability of the neck linker to dock is reliant on residues conserved only in the $\mathrm{Eg} 5$ subfamily [20]. This reversible process is enabled by a cluster of secondary structures that consists of $\alpha 4, \mathrm{~L} 12$ and $\alpha 5$ and is connected to switch II by L11, which in the ATP-bound state adopts a conformation which is amenable to neck-linker docking [17].

\section{Eg5 \& HSET as therapeutic targets Eg5 target validation}

Owing to their roles in cell division, $\mathrm{Eg} 5$ and HSET both represent potential cancer-selective therapeutic targets. Eg5 is reportedly selectively overexpressed in several tumor types, including those of the breast, colon, lung, ovary and uterus [23]. An association of high Eg5 expression and poor clinical outcome has been established in several cancer types including non-muscle invasive bladder urothelial carcinoma, [24] renal cell carcinoma [25] and pancreatic adenocarcinoma [26]. Transgenic mice overexpressing $\mathrm{Eg} 5$ suffer chromosome missegregation, genomic instability and have a higher incidence of tumor formation compared with control animals [27]. Depletion of $\mathrm{Eg} 5$ using endonuclease-prepared siRNA (esiRNA) in HeLa cells distinctively showed perturbation of bipolar spindle formation causing cells to exhibit monopolar spindles, termed 'monoasters' [14]. Similarly, depletion of $\mathrm{Eg} 5$ using alternative methods such as antisense oligomers (ASO) or siRNA in other cell lines decreased cell proliferation and increased apoptotic cell death [28]. Targeting Eg5 with siRNA has been shown to kill tetraploid cells more efficiently than diploid precursor cells, suggesting that clinical Eg5 inhibition may provide a window of selectivity for cancer cells over normal cells, thus minimizing side effects [29]. Depletion of Eg5 


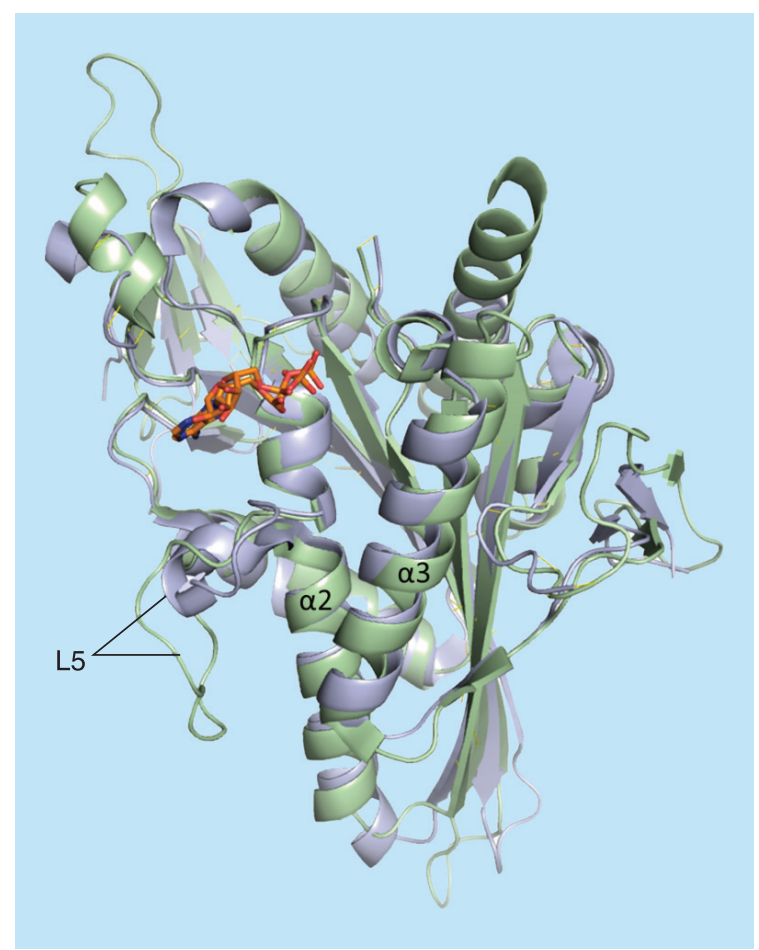

Figure 3. Overlay of crystal structures of Eg5 (PDB 1Il6; light green) and HSET (PDB 2REP; blue). Bound ADP is shown in stick form (orange). Notable differences in the length of loop 5 element are evident.

using ASO treatment in $\mathrm{CaP}$ and $\mathrm{LNCaP}$ prostate cancer cell lines reduced both $\mathrm{Eg} 5 \mathrm{mRNA}$ and $\mathrm{Eg} 5$ protein levels, resulting in dose-dependent growth inhibition, G2/M arrest and apoptosis. Interestingly, low-dose Eg5-ASO seemed to antagonize the cytotoxic effects of paclitaxel. In vivo, ASO monotherapy decreased both $\mathrm{CaP}$ and $\mathrm{LNCaP}$ tumor growth, but combination treatment with paclitaxel did not elicit an additive response [30]. In vivo knockdown of $\mathrm{Eg} 5$ was also effective in UG87 glioblastoma and MDAMB-231 breast cancer [28], ovarian cancer and melanoma [31] and EPP85 pancreatic tumor xenograft models [26].

$\mathrm{Eg} 5$ was found to be highly expressed in blast-crisis chronic myelogenous leukemia (BC-CML) patient samples and cell lines, which were Philadelphia chromosome positive. Inhibition of the Bcr-Abl tyrosine kinase by imatinib was shown to downregulate $\mathrm{Eg} 5$ expression in imatinib-sensitive, but not in imatinibresistant or kinase-negative, cell lines [32]. However, knockdown of $\mathrm{Eg} 5$ using ASO technology induced G2/M arrest and cell death in both imatinib-sensitive and resistant cell lines, suggesting that an Eg5 inhibitor could be used clinically in patients who have developed resistance to Bcr-Abl kinase inhibitors [32]. $\mathrm{Eg} 5$ expression in patients with non-small cell lung cancer (NSCLC) was correlated with cyclin B1 expression and appeared to be predictive of improved clinical response to antimitotic agents in combination with platinum therapy. In this study, $37 \%$ of $\mathrm{Eg} 5$-positive patients showed a clinical response to treatment, compared with only $10 \%$ of Eg 5 -negative patients [33].

\section{HSET target validation}

While esiRNA-mediated depletion of HSET also resulted in perturbation of bipolar spindle formation, the effect was significantly different to that observed following $\mathrm{Eg} 5$ depletion as, in contrast to the distinctive monoaster formation, HeLa cells treated with HSET esiRNA exhibited multipolar spindles [14]. In HeLa cells, HSET siRNA resulted in formation of truncated spindles but did not affect pole formation [15]. However, HSET depletion in breast cancer cell lines indicated that centrosome-amplified cells were particularly sensitive, since they exhibited a higher frequency of multipolar spindles compared with noncentrosome-amplified controls [34]. HSET is believed to play a key role in the survival of centrosome-amplified cancer cells, enabling formation of a pseudo-bipolar spindle through clustering supernumerary centrosomes, which allows the cells to evade apoptotic mechanisms at the mitotic checkpoint [35]. Owing to this role in centrosome clustering, clinical inhibition of HSET may provide a therapy for centrosome-amplified tumors.

HSET siRNA induced multipolar mitoses in breast cancer and melanoma cell lines selectively over nontransformed cells. However, the proportion of cancer cells with multipolarity did not significantly correlate with the percentage of supernumerary centrosomes. Additionally, HSET depletion did not enhance the frequency of supernumerary centrosomes. This data suggested that HSET may be involved in bipolar spindle formation in cancer cells irrespective of centrosome number [36]. A recent study which characterized the expression of HSET in numerous human breast cancer cell lines showed that HSET was highly expressed in all eight tested but was undetectable in human normal mammary epithelial cells. Furthermore, siRNA-mediated knockdown of HSET in two of the cancer cell lines confirmed a reduction in cell viability following treatment [37].

Clinically, HSET overexpression has been correlated with poor prognosis in breast cancer [37] and ovarian adenocarcinoma patients [38]. Elevated HSET gene expression has been detected in numerous other cancer types, including glioblastoma, lung, breast, colon and cervical tumor samples, in comparison to corresponding normal tissues [39]. Additionally, in non-small cell lung cancer, HSET expression was 
Table 1. Structures of Eg5 inhibitors discussed in the text and their biochemical and cellular inhibitory activities.

Compound class Compound identifier Structure

Dihydro-

pyrimidine-thione

(S)-Monastrol 1<smiles>CCOC(=O)C1=C(C)NC(=S)N[C@H]1c1cccc(O)c1</smiles>

rac-Mon-97 2<smiles>CC1=C(C(=O)c2ccccc2)C(c2cccc(O)c2)NC(=S)N1C</smiles>

(S)-Enastron 3<smiles>O=C1CCCC2=C1[C@@H](c1cccc(O)c1)NC(=S)N2</smiles>

(S)-Dimethyl enastron

4<smiles>CC1(C)CC(=O)C2=C(C1)NC(=S)N[C@H]2c1cccc(O)c1</smiles>

(R)-Fluorastrol 5<smiles>CC1=C(C(=O)c2ccc(F)c(F)c2)[C@@H](c2cccc(O)c2)NC(=S)N1C</smiles>

6<smiles>CC(=O)N1CCc2c([nH]c3ccccc23)C1c1cccc(O)c1</smiles>

7

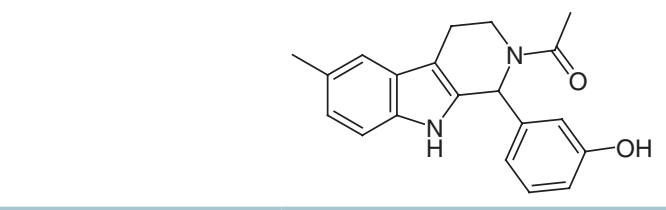

Tetrahydro- $\beta$ carboline

Eg5 biochemical Eg5 cellular $\mathrm{IC}_{50}(\mathrm{nM})^{+}$ $\mathrm{EC}_{50}(\mathrm{nM})^{ \pm}$

30,000

$14,000^{\S}$

[42-44]

2(R) 150

2(R) 1700

2(S) 650

2(S) 6500

$[44,45]$

2000

ND

$[42,44]$

200

ND

$[42,44]$

ND

330

$[44]$

Values are $I_{50}$ in a microtubule-stimulated biochemical assay unless otherwise indicated.

EEffective concentration for $50 \%$ growth inhibition in colon cancer HCT116 cells unless otherwise indicated.

${ }^{5}$ Concentration for $50 \%$ inhibition of microtubule motility in mammalian epithelial kidney BS-C-1 cells.

"Concentration for $50 \%$ induction of mitotic arrest in HeLa cells.

\#MT-stimulated ATPase activity expressed as $\mathrm{Ki}_{\text {ap }}$

${ }^{+\dagger}$ Effective concentration for $50 \%$ growth inhibition due to prolonged mitotic arrest in A-549 human non-small-cell lung cancer cells.

ND: Not determined. 
Table 1. Structures of Eg5 inhibitors discussed in the text and their biochemical and cellular inhibitory activities (cont.).

Compound class Compound identifier Structure

Eg5 biochemical Eg5 cellular

8<smiles>Cc1ccc2[nH]c3c(c2c1)CCN(C(=O)CCN)C3c1cccc(O)c1</smiles>
$\mathrm{IC}_{50}(\mathrm{nM})^{\dagger} \quad \mathrm{EC}_{50}(\mathrm{nM})^{\ddagger}$

9<smiles>Cc1ccc2[nH]c3c(c2c1)CCN(C(=O)CCN)C3c1cccc(F)c1</smiles>

100

ND

[46]

Tritylcysteine

STLC 10<smiles>N[C@@H](CSC(c1ccccc1)(c1ccccc1)c1ccccc1)C(=O)O</smiles>

140

7009

[47]

11<smiles>COc1ccc(C(SC[C@H](N)C(=O)O)(c2ccccc2)c2ccccc2)cc1</smiles>

29

ND

[48]

12<smiles>Cc1ccc(C(CC[C@H](N)C(=O)O)(c2ccccc2)c2ccccc2)cc1C</smiles>

13<smiles>COc1ccc(C2(SC[C@H](N)C(=O)O)c3ccccc3CCc3ccccc32)cc1</smiles>

${ }^{\dagger}$ Values are $\mathrm{IC}_{50} \mathrm{~s}$ in a microtubule-stimulated biochemical assay unless otherwise indicated.

*Effective concentration for $50 \%$ growth inhibition in colon cancer HCT116 cells unless otherwise indicated.

${ }^{5}$ Concentration for $50 \%$ inhibition of microtubule motility in mammalian epithelial kidney BS-C-1 cells.

"Concentration for $50 \%$ induction of mitotic arrest in HeLa cells.

\#MT-stimulated ATPase activity expressed as $\mathrm{Ki}_{\text {a }}$

${ }^{+\dagger}$ Effective concentration for $50 \%$ growth inhibition due to prolonged mitotic arrest in A-549 human non-small-cell lung cancer cells.

ND: Not determined. 
Table 1. Structures of Eg5 inhibitors discussed in the text and their biochemical and cellular inhibitory activities (cont.).

\begin{tabular}{|c|c|c|c|c|}
\hline Compound class & Compound identifier Structure & $\begin{array}{l}\text { Eg5 biochemical } \\
\mathrm{IC}_{50}(\mathrm{nM})^{\dagger}\end{array}$ & $\begin{array}{l}\text { Eg5 cellular } \\
\mathrm{EC}_{50}(\mathrm{nM})^{\ddagger}\end{array}$ & Ref. \\
\hline \multirow[t]{2}{*}{$\begin{array}{l}\text { Thiophene- } \\
\text { containing }\end{array}$} & 14 & 23,000 & $>30,000^{+t}$ & [51] \\
\hline & 16 & 480 & $1000^{+t}$ & [51] \\
\hline \multicolumn{5}{|c|}{$\begin{array}{l}\text { "Values are } I_{5} \mathrm{~s} \text { in a microtubule-stimulated biochemical assay unless otherwise indicated. } \\
\text { "Effective concentration for } 50 \% \text { growth inhibition in colon cancer HCT116 cells unless otherwise indicated. } \\
{ }^{5} \text { Concentration for } 50 \% \text { inhibition of microtubule motility in mammalian epithelial kidney BS-C-1 cells. } \\
\text { "Concentration for } 50 \% \text { induction of mitotic arrest in HeLa cells. } \\
\text { "MT-stimulated ATPase activity expressed as Ki } \\
{ }^{+} \text {Effective concentration for } 50 \% \text { growth inhibition due to prolonged mitotic arrest in A-549 human non-sm } \\
\text { ND: Not determined. }\end{array}$} \\
\hline
\end{tabular}

found to be highly predictive of the presence of brain metastasis in both early and advanced diseases [40].

\section{Eg5 chemical probes: structural findings}

Chemical inhibitors are invaluable tools for the deconvolution of biological processes and validation of novel molecular targets. The two chemical probes monastrol and $(S)$-trityl-L-cysteine (STLC) which specifically target Eg5 have been extensively studied and have provided important information regarding the biological function of this interesting kinesin, as well as structural information and insights into the mechanochemistry of kinesins.

\section{Representative structures of Eg5 inhibitors}

To date, numerous compounds showing inhibitory activity against $\mathrm{Eg} 5$ have been identified, and these have been recently reviewed [41]. In this article, we will focus on the $\mathrm{Eg} 5$ inhibitors with reported crystallographic data on their binding modes (Table 1).

\section{Monastrol \& analogs}

Monastrol was the first chemical probe to be identified as an inhibitor of $\mathrm{Eg} 5$ and was discovered by a phenotypic screening approach described in 1999 [43]. Characterized as an ATP noncompetitive, reversible inhibitor, monastrol was found to induce a 'mono-astral' phenotype in cells, leading to mitotic arrest. Kinetic studies indicated that binding of an inhibitor such as monastrol to the Eg5-ADP complex prevented the force generation and kinesin motility by two modes: first, the release of ADP from the protein is inhibited, preventing completion of the catalytic cycle. Second, the conformational state upon binding has a lower affinity for microtubules, the scaffolding required for bipolar spindle formation [52]. Successful cocrystallization of the inhibitorbound $\mathrm{Eg} 5$ complex in 2004 confirmed the allosteric site of inhibition to be a pocket residing between loop 5 and the $\alpha 2$ and $\alpha 3$ helices of the protein, situated $12 \AA$ away from the nucleotide-binding site (Figure 4A) [53].

The crystal structure of $(S)$-monastrol (1), the more potent of the two enantiomers, bound to Eg5 highlighted a number of key interactions, which are shown in detail in Figure 4B. The majority of $\mathrm{Eg} 5$ inhibitors described to date occupy similar areas within the L5/ $\alpha 2 / \alpha 3$ pocket, including all of those that have reached clinical trials.

A structurally related analog of monastrol bound to the same allosteric site, but the opposite enantiomer, $(R)$-Mon-97 $2(\boldsymbol{R})$, showed higher affinity and thus preferentially cocrystallized in the allosteric site of ADP-bound Eg5. (R)-Mon-97 differs from monastrol 


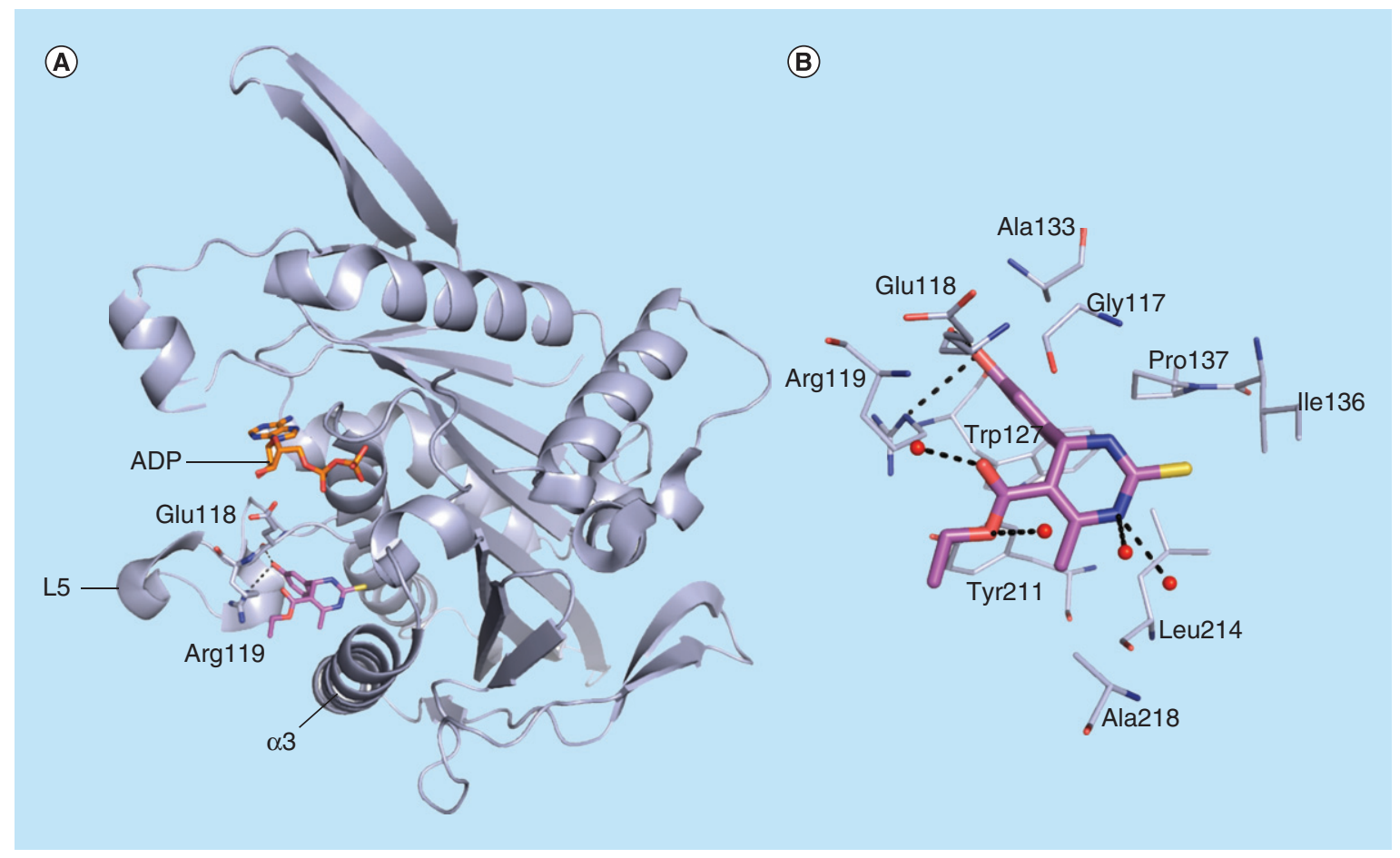

Figure 4. Binding site of monastrol (shown in magenta sticks) (PDB 1X88), located in the L5/ $\alpha 2 / \alpha 3$ allosteric pocket of ADP-bound Eg5. (A) Hydrogen bonds between the phenolic OH and Glu118 (2.7 $⿱$ ) and Arg119 (3.3 $⿱$ ) are shown. Bound ADP is shown in stick form (orange). (B) Detailed binding site view of monastrol bound in the $L 5 / \alpha 2 / \alpha 3$ allosteric pocket. The dihydropyrimidinethione core of monastrol makes several hydrophobic interactions with a surface consisting of the side chains of residues Gly117, Ile136, Pro137, Tyr211, Leu214 and Ala218. A second hydrophobic cleft made up of Arg119, Trp127, Ala133 and Tyr211 houses the phenol ring. Key hydrogen bonds are shown between the phenolic $\mathrm{OH}$ and the carbonyl of the backbone of Glu118, and between the oxygen of the phenol and the guanidine side chain of Arg119. Additional hydrogen-bonding interactions made with water molecules (red) via the ester motif and $\mathrm{NH}$ of the dihydropyrimidinethione core are also shown.

in that the ethyl ester is replaced with a bulky acetophenone group, and one of the nitrogen atoms of the dihydropyrimidinethione motif is methylated. These subtle structural changes resulted in a 'flipped' binding orientation which placed the lipophilic phenyl ring of the acetophenone group in the hydrophobic cleft previously occupied by the dihydropyrimidine core of monastrol, and the thioxo group pointing toward the solvent-exposed surface, making a network of hydrogen-bond interactions with two water molecules [44]. Interestingly, the $(S)$-enantiomer $\mathbf{2}(\boldsymbol{S})$ was also reasonably potent against $\mathrm{Eg} 5$ which warns against the assumption that a single enantiomer will be solely responsible for on-target potency across a given chemical series binding in this site, possibly because the protein is amenable to significant conformational changes. Remarkably, despite the differences in binding mode, the overall protein structures of $\mathbf{1}$ - and $\mathbf{2}(\boldsymbol{R})$-bound $\mathrm{Eg} 5$ are very similar, resulting in identical modes of action (Figure 5A) [45].

Efforts to improve the biochemical and cellular potency of dihydropyrimidinethione-based inhibitors resulted in the discovery of enastron (3), dimethylenastron (4) and fluorastrol (5). Crystallographic studies revealed a similar phenomenon with these structurally related dihydropyrimidinethiones, whereby enastron and dimethylenastron bind primarily in the $(S)$-configuration (Figure $5 \mathrm{~B}$ ) while the $(R)$-enantiomer of fluorastrol binds preferentially. The 3-phenol group is common to all three inhibitors and was shown to be in the same position in all three crystal structures. The additional potency gains in enastron and dimethylenastron were attributed to the reduced flexibility of the molecules, and a better fit into the solvent exposed subpocket of Eg5. Despite pointing toward solvent, the dimethyl groups of dimethylenastron maintain hydrophobic contacts with the main chain of Ala218, and significantly, one methyl group makes a $\mathrm{CH}-\pi$ interaction with Tyr211. The fivefold increased cellular potency of $(R)$-fluorastrol relative to $(R)$-Mon-97 in terms of growth inhibition in an HCT116 cell line (see Table 1) has been attributed to the ability of one of the fluorine atoms to form multipolar interactions within the binding site. Additionally, the electron-rich fluorine atoms are in close proxim- 
ity to a positively charged Arg221 residue, and the resulting electron-deficient aromatic ring that makes a $\pi$-stacking interaction with the salt bridge formed by Glu116 and Arg221 is more favorably positioned near to the negatively charged carboxylate of Glu116, which could be partly responsible for the potency gains observed in this assay [44]. However, since no biochemical assay data are available for $(R)$-fluorastrol, it is not possible to determine whether all of the observed cellular potency gains are a direct result of improved ligand-protein interactions. Other aspects such as differences in cell permeability as a result of increased inhibitor lipophilicity, and equilibria between different conformational states of $\mathrm{Eg} 5$ could also be contributing to the differences in cell potency. The flexibility of $\mathrm{Eg} 5$ to accommodate structurally related compounds in opposite binding modes highlights the caveats in using in silico drugdesign predictive tools against highly mobile proteins such as kinesins.

While tetrahydro- $\beta$-carboline inhibitors (Table 1, compounds 6-9) were discovered independently in a high-throughput screening campaign and are a structurally distinct compound class, these inhibitors were found to occupy the same binding region as the dihydropyrimidinethione-based analogs [45].
Hit compound $\mathbf{6}$ was identified as a modestly potent inhibitor of $\mathrm{Eg} 5$ and incorporation of a methyl group to give 7 improved potency tenfold, presumably by increasing hydrophobic contacts in the lipophilic Leu214 region. While the compounds were screened as racemates, the $(R)$-enantiomer of 7 bound preferentially to $\mathrm{Eg} 5$ (PDB 3K3B, not shown), and the crystal structure revealed that the phenolic motif makes a hydrogen-bonding interaction with Glu118 (2.6 $\AA$ ), thus mimicking the $\mathrm{OH}$ group of monastrol. A second hydrogen bond is evident between the $\mathrm{NH}$ of the tetrahydro- $\beta$-carboline core and Glu116 $(3.1 \AA)$ and a third is identified between the amide carbonyl lone pair and the main chain amide $\mathrm{NH}$ of Arg119, although with suboptimal geometry. The potency of 7 was increased further by incorporating a basic side chain (8), which was hypothesized to interact with Glu215 or Tyr211, although not crystallographically confirmed. Interestingly, the phenol motif which presents a metabolic liability for further development of this series could be successfully replaced with a fluorophenyl group (9) without significantly compromising potency [46]. Since a structure of 9 bound to $\operatorname{Eg} 5$ has not been reported, the reasons why this compound retains a high level of potency remain unclear. An alternative binding ori-

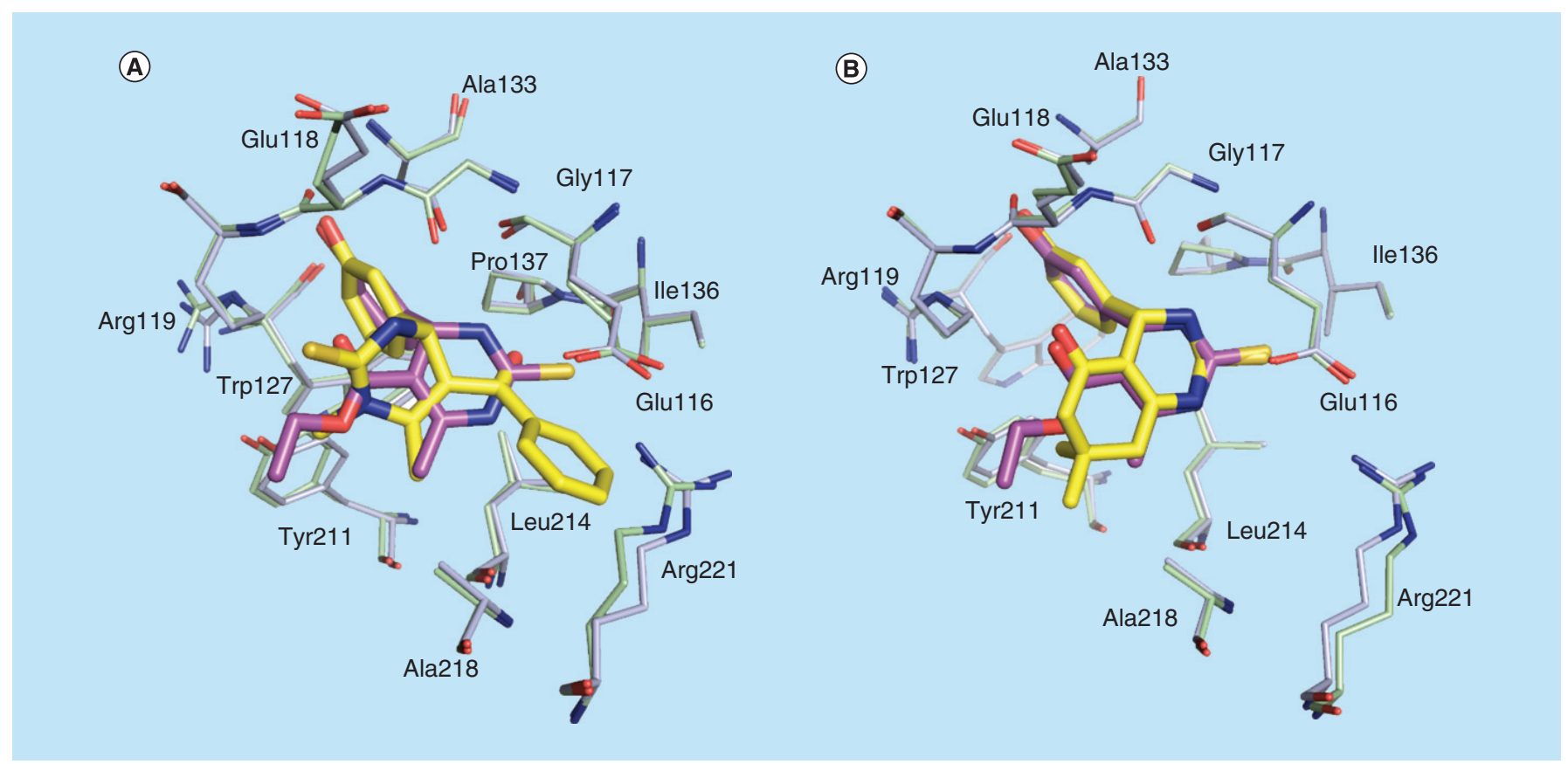

Figure 5. Binding of monastrol analogs to Eg5. (A) Overlay of monastrol (1) (magenta, PDB 1X88, light blue ADP-Eg5) and (R)Mon-97, 2(R) (yellow, PDB 2IEH, pale green ADP-Eg5) showing the 'flipped' binding orientation. Hydrogen bonds between the phenolic $\mathrm{OH}$ of $2(\mathrm{R})$ and the backbone of Glu118 (2.6 $⿱$ ) and a weaker interaction with the side chain of Arg119 (3.4 $\AA$ ) analogous to those formed by the phenolic $\mathrm{OH}$ of monastrol exist but are not shown. An additional hydrogen bond between the $\mathrm{NH}$ of the dihydropyrimidinethione core and Gly117 (2.6 ̊) is also formed. (B) Overlay of monastrol (1) (magenta, PDB 1X88, light blue ADP-Eg5) and (S)-dimethylenastron (4) (yellow, PDB 2X7D, pale green ADP-Eg5). The two ligands bind in the same orientation and hydrogen bonds to the protein are identical. 
entation as seen with the dihydropyrimidine-based analogs, or a pseudo hydrogen-bonding interaction between the fluorine atom and the key Glu118 of Eg5 could be possible explanations.

\section{(S)-Trityl-L-cysteine (STLC) \& analogs}

STLC (10) was identified as an ATP-noncompetitive and reversible inhibitor of $\mathrm{Eg} 5$ in both basal and microtubule-stimulated biochemical assays. Additionally, STLC caused mitotic arrest in HeLa cells. The binding site of STLC is the same as that of monastrol, occupying the L5/ $\alpha 2 / \alpha 3$ allosteric pocket. However, STLC had much tighter binding than monastrol, owing to an eightfold faster association rate and fourfold slower release rate [47]. Crystallographic studies showed that STLC makes a number of hydrophobic, $\pi$-stacking and hydrogen-bonding interactions with Eg5 (Figure 6). Most strikingly, not only were the interactions between STLC and $\mathrm{Eg} 5$ revealed from the cocrystallization studies, but the sequence of $\mathrm{Eg} 5$ conformational changes upon inhibitor binding was elucidated, as an intermediate state of the kinesin-inhibitor complex was serendipitously captured [54].

Opportunities for the optimization of STLCbased inhibitors have been exploited to improve upon biochemical, cellular and in vivo activity.
Incorporation of small substituents at one or more positions on the rotationally interchangeable phenyl rings increased potency by maximizing hydrophobic interactions in the core of the protein, for example, $(S)$-methoxytrityl-L-cysteine (11), while bulkier hydrophobic substituents were not productive as the trityl group rotates to place the larger substituent in the solvent-exposed subpocket [48,55-56]. Removal of the metabolically labile sulfur atom and isosteric replacement with a carbon linkage gave a series of triphenylbutanamines [49,57] exemplified by compound 12 which showed significant tumor growth inhibition in a range of cancer cell lines (HCT116, LNCaP, K562, PC3, BxPC-3 and NCI-HI299) and gave regressions of a subcutaneous tumor xenograft of LXFS538 lung cancer cells following an intraperitoneal dosing schedule [49].

More recently, conformationally restrained tricyclic analogs of STLC have been reported, which sought to increase the hydrophobic van der Waals interactions observed between STLC and the protein while reducing entropy. Indeed, ring closure of $(S)$-methoxytrityl-L-cysteine with an ethylene linker to give a seven-membered fused carbocycle (13) displayed a 12 -fold improvement in biochemical potency and an eightfold improvement in cytotoxicity in the

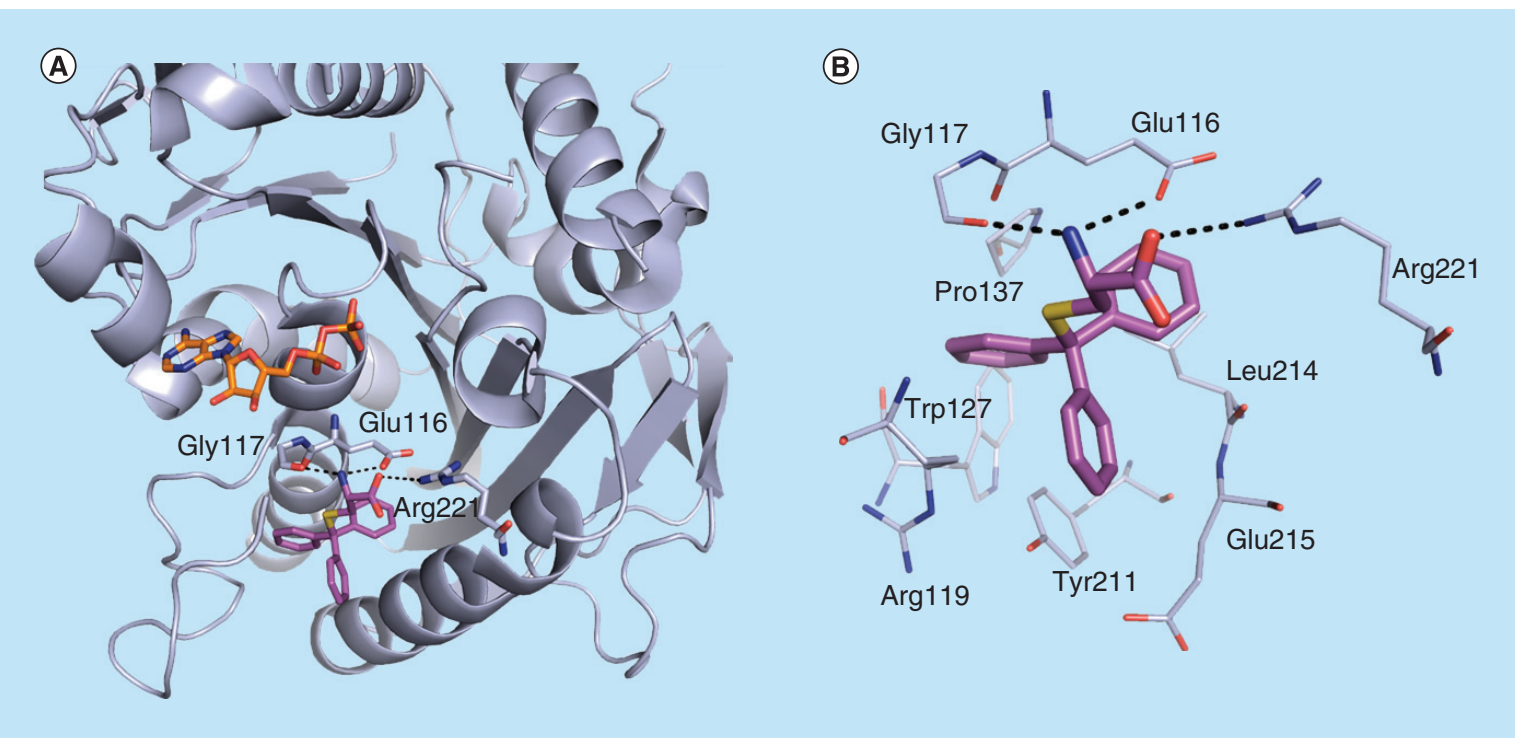

Figure 6. The binding interactions of (S)-trityl-L-cysteine (magenta, PDB 3KEN) with ADP-Eg5 (light blue).

(A) Polar interactions are made between the amine of STLC with the side chain of Glu116 (3.2 A) and the main chain of Gly117 (2.7 $\AA$ ), and the acid of STLC forms a hydrogen bond with Arg221 (3.6 ̊) (dotted lines). Bound ADP is shown in stick form (orange). (B) Detailed binding site view of STLC bound in the L5/ $22 / \alpha 3$ allosteric pocket. The lipophilic triad of phenyl rings in STLC makes several hydrophobic interactions with the alkyl side chains of residues Glu215, Glu116 and Arg119, anchoring the compound into the binding site. Several aromaticstacking interactions are evident from the crystal structure including an edge-to-face interaction with Trp127 and a $\mathrm{CH}-\pi$ interaction between Pro137 and the first phenyl ring. The second phenyl ring makes an offset stacked $\pi-\pi$ interaction with Tyr211, and the third aromatic ring makes another $\mathrm{CH}-\pi$-type interaction with the aliphatic side chain of Leu214. The polar side chain of STLC is exposed to solvent, making hydrogen bonds between the residues (shown) and water molecules (not shown). 


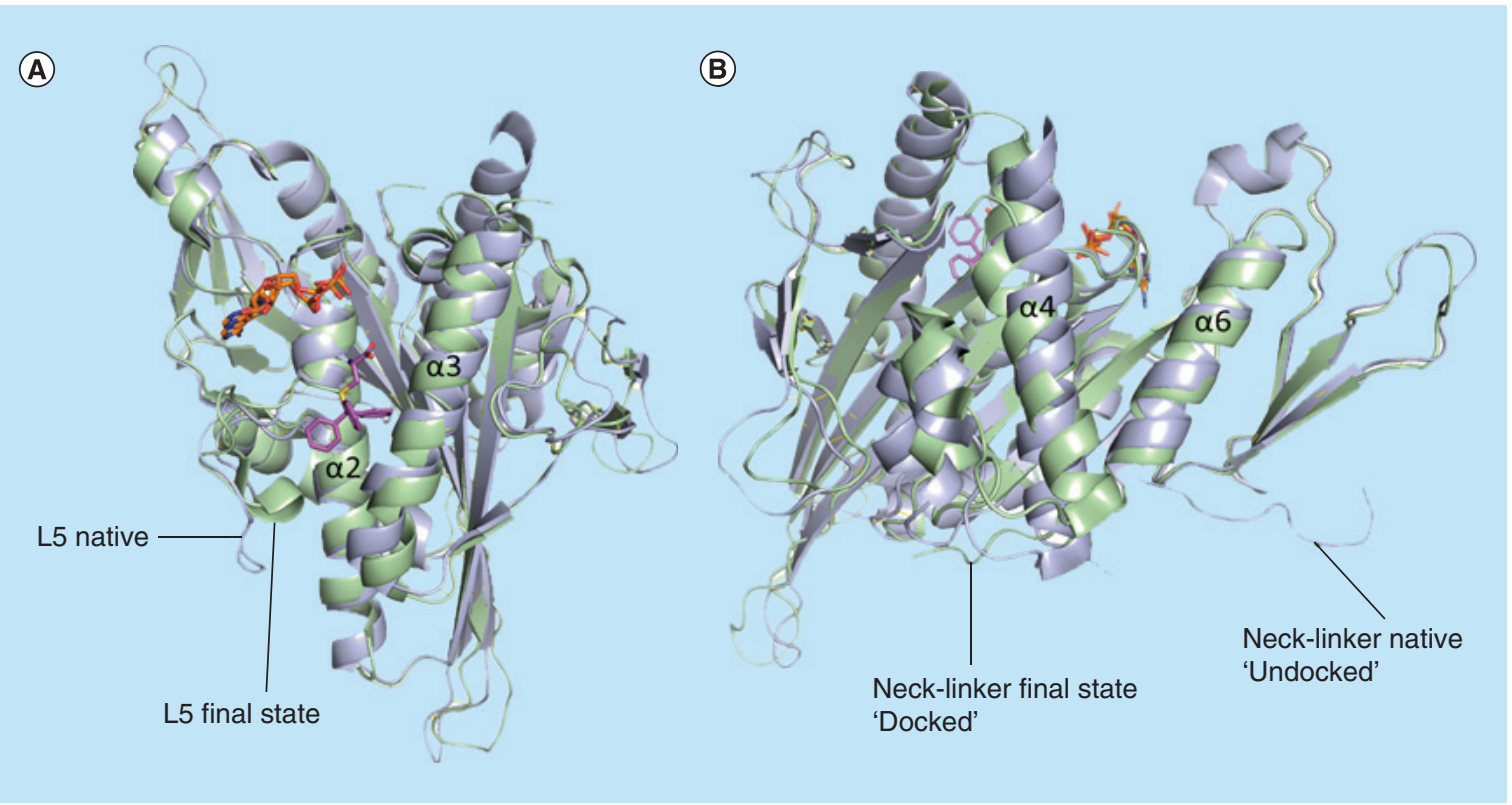

Figure 7. The sequence of conformational changes induced upon inhibitor binding to Eg5. Overlays of native ADP-Eg5 (light blue, PDB 1II6) with the crystal structure of STLC-ADP-Eg5 in its final inhibitor-bound state (pale green, PDB 2WOG). Bound ADPs (orange) and STLC (magenta) are shown in stick form. (A) Loop L5 in native Eg5 swings round to close the inhibitor-binding pocket, and helix $\alpha 3$ has shifted. (B) The switch II cluster (helix $\alpha 4$, loop L12 and helix $\alpha 5$ ) is shifted to a 'permissive' conformation which has allowed the neck linker to dock onto the microtubule-binding face of Eg5.

HCT116 cell line. In vivo activity in an HCT116 colon cancer xenograft model following a $25 \mathrm{mg} / \mathrm{kg}$ intravenous dose was also reported [50].

\section{The pathway of Eg5 structural changes elucidated using chemical tools}

The binding of an Eg5 inhibitor results in a wide variety of drug-induced structural changes, and elucidation of these effects has been of considerable interest. While crystallographic snapshots of monastrol and its analogs bound to $\mathrm{Eg} 5$ provided insight into the mode of action of inhibition, how these changes were initiated and how subsequent conformational changes were connected remained unclear.

Studies using Forster resonance energy transfer (FRET) indicated a biphasic conformational change of the Eg5 motor domain. The first phase occurs rapidly and involves movements within the inhibitor-binding site, including the residue Trp127 which was monitored for quenching by monastrol binding. The second much slower phase seemed to result in the docking of the neck linker onto the motor domain [58,59]. The crystallographically determined intermediate state of STLC-bound Eg5 corroborated the conclusions drawn from FRET studies on the intermediate transition states involved (Figure 7A \& B), confirming that binding of STLC causes drug-induced transition through three distinct stages: First, the loop L5 in native Eg5 swings round to close the inhibitor-binding pocket. Second, the switch II cluster (helix $\alpha 4$, Loop L12 and helix $\alpha 5$ ) rearranges to adopt a 'permissive' conformation, opening up space for the final stage. Third, the neck-linker docks onto the motor domain to give the final inhibitor-bound complex [54]. As Eg5 has a unique, extended loop 5, it is currently unknown whether kinesins with shorter L5 motifs such as HSET exhibit similar conformational changes upon inhibition.

\section{HSET inhibitors as chemical tools CW069}

While previously considered a less tractable target than $\mathrm{Eg} 5$, the recent emergence of two selective chemical probes for this motor protein has provided exciting opportunities to address the potential role for HSET inhibitors in cancer therapy (Table 2). In the absence of any available structurally characterized chemical tools, CW069 (17) was identified using a 'chemogenomics-based' compound selection approach, using the principle that similar proteins will bind to similar ligands [60-62]. An in silico model for HSET binding was developed, based on the existing inhibitors of $\mathrm{Eg} 5$ mined from the CHEMBL database. Owing to the high degree of sequence similarity $(80 \%)$ in the motor domains of $\mathrm{Eg} 5$ and HSET, it was rationalized that inhibitors of HSETbinding $L 5 / \alpha 2 / \alpha 3$ would be identified using this approach. Biochemical profiling of a triaged selection of 50 compounds led to the discovery of two 
Table 2. Published selective inhibitors of HSET and their biological activities.

Compound identifier

inhibitors, one of which, containing a $\gamma$-lactone benzoic acid moiety, was selective for HSET over $\mathrm{Eg} 5$. Hit expansion led to the discovery of CW069, which inhibits HSET selectively with modest biochemical potency. Of 64 analogs tested, only CW069 displayed any activity against HSET, indicating a narrow structure-activity relationship window for this chemotype.

A ligand-based-binding mode prediction (using PDB files 2REP and 1 I16 with CW069 plus one other analog) gave calculated protein-ligand interaction enthalpic energies for HSET and Eg5, which were consistent with the selectivity observed against Eg5. The model predicted a key hydrogen-bond interaction between Arg521 and the carboxylate of CW069, which appeared to be responsible for the selectivity observed between HSET and Eg5, where the analogous residue is Ala218. Additional proposed interactions included: hydrogenbond interactions between backbone $\mathrm{CO}$ and $\mathrm{NH}$ of Gly423, Leu517 and the carboxylate and amine groups of CW069. Interestingly, a structurally similar analog of CW069 was not HSET selective, which was rationalized using molecular dynamics data for the protein. Despite the shorter chain length of loop 5 in HSET relative to $\mathrm{Eg} 5$, HSET L5 was found to be extremely dynamic, particularly around a glycine-rich motif, which is not present in Eg5. This resulted in opening of the binding pocket, allowing CW069 to be accommodated, whereas Eg5 consistently showed partial closure of the analogous-binding space. Despite its weak biochemical activity, CW069 was shown to increase multipolar spindle formation in breast cancer cell lines con- taining supernumerary centrosomes (MDA-MB-231 and BT549) without altering bipolar spindle formation in a noncentrosome-amplified cell line [60].

\section{AZ82}

High-throughput screening of 800,000 compounds followed by subsequent iterative medicinal chemistry was used to identify the potent HSET inhibitor AZ82 (18). Impressively, over 1500 compounds were profiled within 1 week using an integrated HTS, synthesis and screening campaign to enable rapid optimization of the chemical series. An HSET selective inhibitor with no activity against a panel of nine other kinesins including Eg5, AZ82 bound to the HSET/microtubule binary complex and inhibited microtubule-stimulated HSET ATPase activity. Co-sedimentation analysis revealed AZ82 to be ATP/ADP competitive, stabilizing an HSET state with a higher affinity for microtubules, an opposite mode of action from the $\mathrm{Eg} 5$ inhibitor monastrol which inhibits ADP release from the protein and results in an $\mathrm{Eg} 5$ conformational state with a lower binding affinity for microtubules. Equilibrium dialysis/mass spectrometry (ED/MS) and fluorescent nucleotide exchange experiments also suggested that AZ82 bound to the HSET/microtubule complex, not HSET or microtubules alone [63,64].

Unfortunately, no crystal structure of HSET with an inhibitor bound has yet been solved, and ligand-protein complex crystallography was noted to prove difficult in the case of AZ82 despite its high affinity for the target. This is possibly due to the requirement for HSET to be in the microtubule-bound state to bind AZ82. In order 
to gain insights into the binding mode of this inhibitor, a homology model based on available kinesin-inhibitor structures was constructed. Owing to the structural similarity of AZ82 to GSK923295, a compound which binds in the analogous $\mathrm{L} 5 / \alpha 2 / \alpha 3$ allosteric-binding pocket of the structurally related kinesin CENP-E, the model incorporated aspects of the inhibited CENP-E protein [65] as well as the crystal structures of $\mathrm{Eg} 5$ bound to the clinical candidate EMD-534085 [66] and ADPbound HSET. The homology model suggested that the thiophene ring was buried deeply in the hydrophobic pocket lined with aromatic rings of Tyr461, Phe542 and the aliphatic chain of Glu421. The trifluoromethyl moiety of the biaryl group was positioned in a pocket formed by the L5 loop, and the polar pyrrolidine tail was directed toward the nucleotide-binding site $[63,64]$. The ability of AZ82 to reverse the monopolar spindle phenotype observed following treatment with an $\mathrm{Eg} 5$ inhibitor and cause centrosome declustering in centrosome-amplified cancer cell lines provided further evidence that inhibition of HSET presents an alternative opportunity for pharmacological modulation of the motor protein function.

\section{Eg5 inhibitor clinical candidates}

The Eg 5 inhibitors which have been evaluated in clinical trials to date are ATP noncompetitive and target the L5/ $\alpha 2 / \alpha 3$ allosteric pocket (Table 3). Despite nine inhibitors reaching the clinic, all are based around only three broad chemical scaffolds with several overlapping structural features (although the structure of 4SC-205 is undisclosed). Since these clinical candidates target the same binding site as that of monastrol and STLC, a common mode of inhibition is shared. The discovery and development of Eg5 inhibitors has been reviewed in detail elsewhere [41,67-68] and therefore this section will summarize the key features of the binding modes and the clinical outcomes to date.

\section{Binding features of Eg5 clinical candidates}

Despite the fact that ispinesib (19) was the first to reach the clinic, and the quinazolinone-based scaffold of this inhibitor dominates current clinical candidates, little is reported regarding the first discovery of this chemotype and its subsequent development. A family of quinazolinone-containing analogs was identified following a high-throughput screening campaign, which displayed promising initial properties including $\mathrm{IC}_{50}$ values below $1 \mu \mathrm{M}$, as well as sub-10 $\mu \mathrm{M}$ in vitro potency in cells and greater than 100 -fold selectivity over a panel of 12 other kinesins. Key areas of optimization for both potency and cell permeability included the length and basicity of the ethylamine side chain, the requirement for 4-substitution on the benzamide group and the required substi- tution and stereochemistry at the $\alpha$-amino group. The resulting candidate compound ispinesib had $\mathrm{Ki}<1 \mathrm{nM}$, $\mathrm{GI}_{50}<1 \mathrm{nM}$ in the SKOV3 human ovarian carcinoma cell line, good aqueous solubility and oral bioavailability [70]. Many of the structural features of ispinesib are present in the second-generation inhibitor SB-743921 (20) with the core quinazolinone heterocycle being replaced by a chromenone. This subtle change improved activity against $\mathrm{Eg} 5$ giving a remarkably potent inhibitor $(\mathrm{Ki}=0.1 \mathrm{nM})$ with dose-dependent activity in a number of tumor xenograft models [71]. Other investigations led to the discovery of AZD-4877 $\left(\mathbf{2 1}, \mathrm{IC}_{50}=\right.$ $2 \mathrm{nM}$ ), also in clinical development, where the quinazoline is replaced by an isothiazolo[5,4-d]pyrimidin4-one [72]. While the quinazolinone Arq621 (22) has more structurally diverse substituents, many of the key features of ispinesib are retained. There is no crystallographic data reported for Arq621 at present, but it is assumed that the binding mode will be similar to that of ispinesib [73]. The majority of ispinesib's potency is likely due to the extensive hydrophobic interactions, inducing a larger pocket than that of monastrol (Figure 8A) [74,75]. Remarkably, only one direct hydrogen-bonding interaction is consistently made with the protein, as it is observed in three of the four chains within the ternary complex. In the fourth chain of the ternary complex, the aminopropyl side chain is disordered, highlighting its flexibility.

High-throughput screening has proven to be a successful technique for the identification of $\mathrm{Eg} 5$ inhibitor scaffolds with subsequent optimization leading to the discovery of the other clinical candidates; hexahydro-2H-pyrano[3,2-c] quinolone (HHPQ) compound EMD-534085 (23) [66] and the 2,5-dihydropyrrole MK-0731 (24) [77,78]. The clinical candidates ARRY-520, 25 [79] and LY2523355, 26 [80] bear structural resemblance to MK-0731, but replace the 2,5-dihydropyrrole core with a 1,3,4-thiadiazoline motif. Despite the structural diversity between EMD-534085, MK-0731 and ispinesib, crystallography revealed several overlapping features (Figure 8B). The thiophene-containing chemical probes 14, 15 and 16 (Table 1) also bind similarly to MK-0731 and were identified via a HTS approach [51].

The C-9 trifluoromethyl substituent of EMD534085 also occupies a position analogous to the chloroquinazolinone moiety of ispinesib, but the basic side chain of EMD-534085 serves to increase aqueous solubility of the lipophilic core, pointing toward solvent. This improves the metabolic stability and pharmacokinetic properties of the lipophilic core but is not reported to make any binding interactions with the protein. Additionally, a putative HHPQ-ispinesib hybrid compound (27) was eight- 
fold less active than EMD-534085, supporting the differences in binding modes (Figure 9) [66].

\section{Clinical outcomes with Eg5 inhibitors}

Despite all Eg5 inhibitors reaching clinical trials displaying low nanomolar biochemical potency and good cel- lular activity against the target, clinical data have been disappointing to date, as many $\mathrm{Eg} 5$ inhibitors have failed to show efficacy as a monotherapy. One of the exceptions is ARRY-520 (25) (filanesib), which has demonstrated clinical activity in patients with relapsed or refractory multiple myeloma [81]. According to the product pipe-

\section{Table 3. Clinical evaluation of Eg5 inhibitors.}

\section{Compound identifiers/}

Structure

Phase

Clinical trial status ${ }^{\dagger}$

company

Ispinesib (SB715992) 19

Cytokinetics

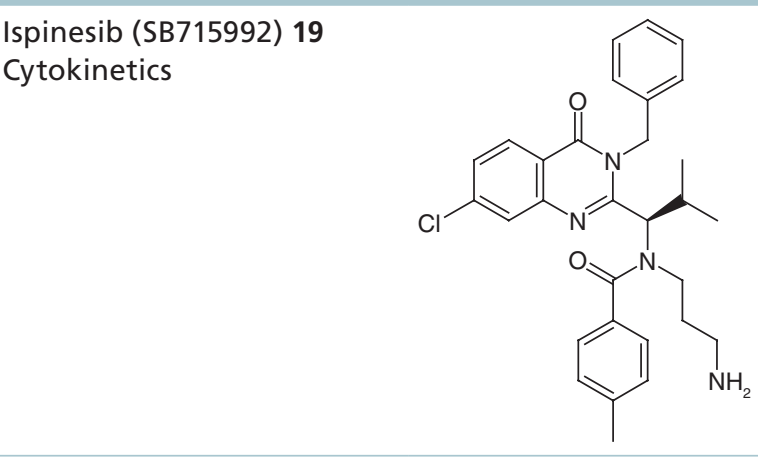

\section{I/II}

15 trials completed

1 trial terminated

SB743921 20

Cytokinetics

AZD4877 21

AstraZeneca<smiles>Cc1ccc(C(=O)N(CCCN)[C@@H](c2oc3cc(Cl)ccc3c(=O)c2Cc2ccccc2)C(C)C)cc1</smiles><smiles>Cc1ccc(C(=O)N(CCCN)[C@H](c2nc3snc(C)c3c(=O)n2Cc2ccccc2)C(C)C)cc1</smiles>

Arq621 22 ArQule
I/II

I/II
2 trials completed
3 trials completed

3 trials terminated<smiles>C#CC[C@@H](N(CCCN)C(=O)c1cccc(Cl)c1F)n1cnc2cc(Cl)ccc2c1=O</smiles> 


\section{Table 3. Clinical evaluation of Eg5 inhibitors (cont.).}

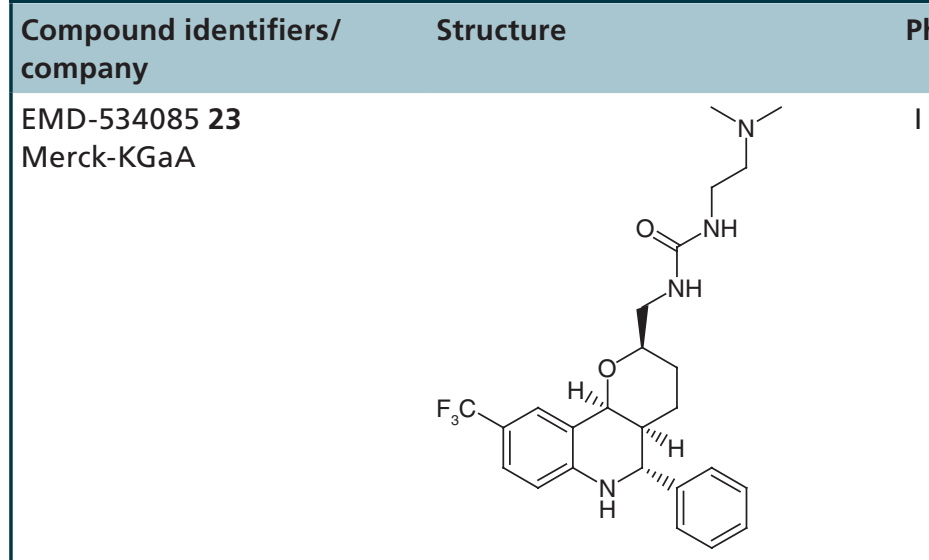

Clinical trial status ${ }^{\dagger}$

MK-0731 24 Merck \& Co

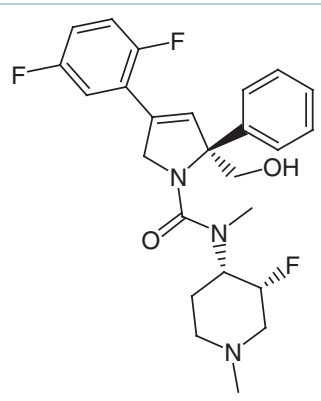

1 trial completed

\section{Not known ${ }^{\ddagger}$}

ARRY-520 (Filanesib) 25

Array BioPharma

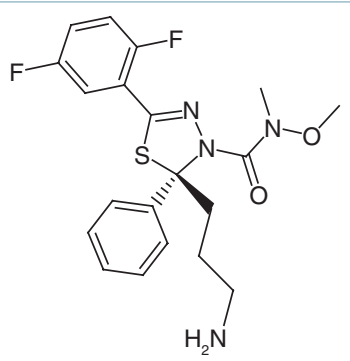

2 trials completed

4 trials ongoing

2 trials planned

\section{SB743921 20}

Cytokinetics

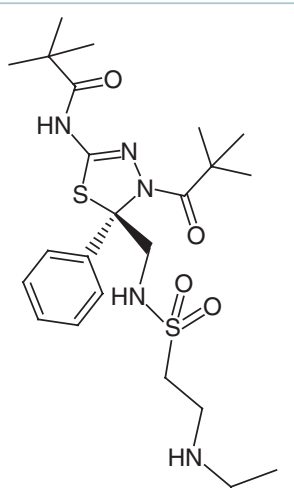

I/II

2 trials completed

4SC-205 4SC AG

Not disclosed

I

1 trial completed

'Data from ClinicalTrials.gov.

The compound is reported to have entered clinical trials $[8,69]$.

lines available on the relevant pharmaceutical company websites (accessed October 2015), only two compounds appeared to be reported as in clinical development: ARRY-520 and 4SC-205 (Table 3). The reasons for the poor activity of many $\mathrm{Eg} 5$ inhibitors in the clinic are unclear, although several hypotheses have been proposed. It has been postulated that the clinical effectiveness of classical microtubule-targeted agents in general may be due in part to the disruption of the microtubuleassociated functions of nondividing tumor cells [82], 
(A)

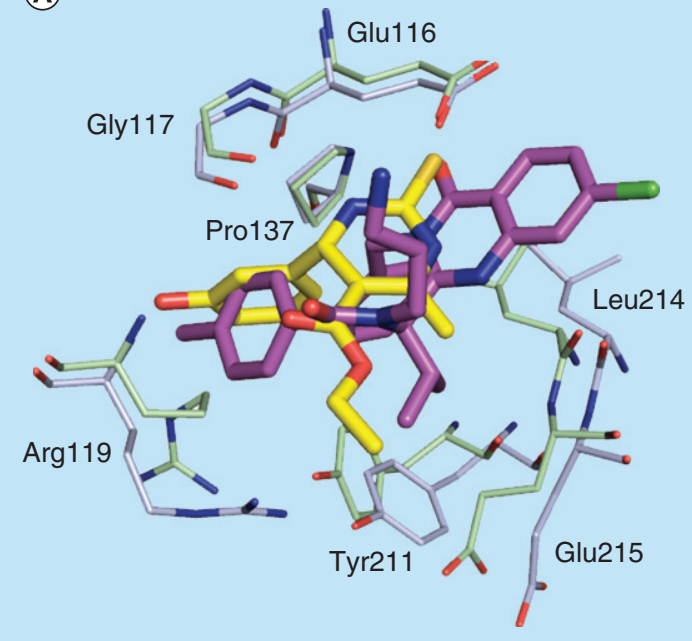

(B)

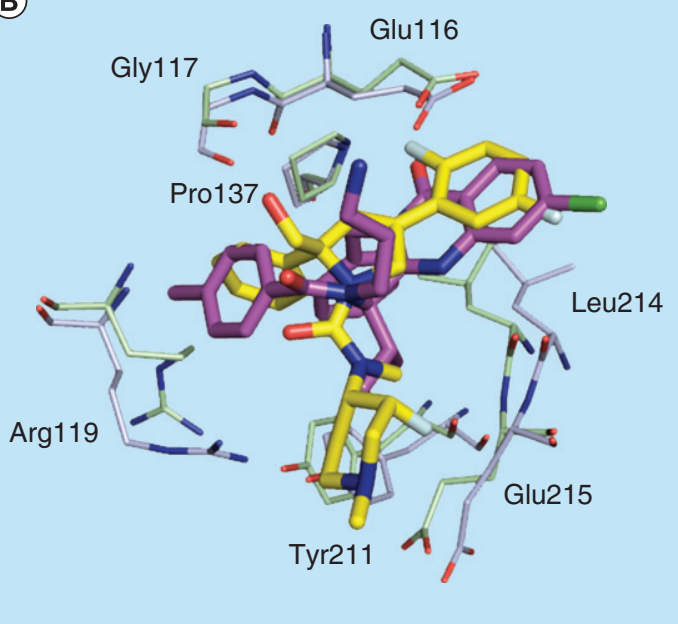

Figure 8. Binding of the clinical candidates ispinesib and MK-0731 to Eg5. (A) Overlay of the binding of ispinesib (19) (magenta ligand, light blue ADP-Eg5, PDB 4AP0) and monastrol, 1 (yellow ligand, pale green ADP-Eg5, PDB 1X88). Ispinesib induces a larger binding pocket than monastrol, owing to a $1.3 \AA$ shift of the Leu214 backbone to accommodate the benzyl group, which makes stacking interactions with Pro137 and Tyr211. Tyr211 and Glu215 are also shifted by 1-2 A relative to monastrol to accommodate both the benzyl group and the aromatic ring of the benzamide moiety. The methyl benzamide group mimics the phenol ring of monastrol, almost overlapping, but the side chain of Arg119 must shift over $3 \AA$ to accommodate this group. The flexible alkylamino chain in ispinesib forms a network of hydrogen bonds by directly interacting with the carboxylate of Glu116, which in turn makes a hydrogen bond with Gly117 via a water molecule (not shown). (B) Overlay of the binding of ispinesib (19) (magenta ligand, light blue ADP-Eg5, PDB 4AP0) and the clinical candidate MK-0731 (24) (yellow ligand, pale green ADP-Eg5, PDB 3CJO). The difluorophenyl motif of MK-0731 overlays well with the chloroquinazolinone core of ispinesib but MK-0731 makes an interaction with Gly117 rather than Glu116. While the basic side chain of Arg119 is pushed aside upon ispinesib binding, it is able to fold atop MK-0731, making a favorable $\pi$-stacking interaction in doing so $[75,76]$.

or through inhibition of angiogenesis by host vascular cells [83]. Eg5 is almost exclusively expressed during the mitotic phase of the cell cycle, although some is expressed in G2. As a result, drugs targeting Eg5 may only have a therapeutic effect on cells which are undergoing cell division at the time of treatment [84]. Since preclinical models based on tumor xenografts have faster cell-cycling rates, the preclinical results may not be sufficiently realistic as predictors of clinical success [49]. This could also apply to the development of inhibitors of other kinesins whose expression is limited to M-phase. Interestingly, recent clinical data using the oral Eg5 inhibitor 4SC-205 indicated that a continuous dosing scheme of $20 \mathrm{mg}$ per patient, once daily could overcome this 'proliferation rate paradox'. A clinical response (stable disease) was observed in $67 \%$ of patients for more than 100 days undergoing this regimen [85].

A second challenge to clinical efficacy is possible functional redundancy among mitotic kinesins. Primarily involved in maintenance of the bipolar spindle [8], KIF15 (kinesin-12) has been proposed as a potential compensatory kinesin for $\mathrm{Eg} 5$. Evidence to suggest that KIF15 drives bipolar spindle assembly in the absence of $\mathrm{Eg} 5$, compensating for its inhibition when overexpressed, has been reported [11]. Interest in this functionally related kinesin has led to its biochemical characterization and comparison to $\mathrm{Eg} 5$. In contrast to Eg5, KIF15 does not contain a second nucleotide-independent microtubule-binding site in its C-terminal tail and operates as a dimer to cross-link kinetochore microtubules, as opposed to the homotetrameric structure of Eg5. Owing to the structural differences between the two kinesins, microtubule binding is most likely carried out by the targeting protein for Xklp2 (TPX2), which complexes to KIF15 through its C-terminus, promoting centrosome separation. Interestingly, TPX2 is also known to interact with the mitotic kinase Aurora A, and the sensitivity of nonHodgkin lymphoma (NHL) cell lines to the Aurora A inhibitor MK-8745 was increased when TPX2 was depleted with siRNA [86,87]. It has therefore been proposed that KIF15 and its protein-protein interaction with TPX2 represent interesting therapeutic targets, perhaps with utility in combination with an $\mathrm{Eg} 5$ inhibitor [11,88-89]. Clinical use of drug combinations is a valid chemotherapeutic strategy, which has been shown to result in synergistic effects in some cases, and 
offers a greater chance of overcoming acquired drug resistance [90]. The effectiveness of the $\mathrm{Eg} 5$ candidate SB-743921 was enhanced when dosed in combination with the Aurora-A kinase inhibitor alisertib and restored activity in a drug-resistant cell line [91].

A third, mechanochemical, explanation for resistance to $\mathrm{Eg} 5$ inhibition is proposed. It has been demonstrated that the flexible loop L5 which is coupled to the nucleotide-binding site and the neck-linker element which initiates forward motility, serve to accelerate ADP release during the initial microtubule-binding event of the catalytic cycle. However, the observation that a seven amino acid deletion within L5 can still hydrolyze ATP suggests that L5 is not essential to promote subsequent movement along the microtubules. While inhibitors targeting the $\mathrm{L} 5 / \alpha 2 / \alpha 3$ allosteric site are known to kinetically slow the release of ADP, doubts have arisen regarding how effectively mitosis can be inhibited by these slowed but nonarrested motors, particularly since multiple motors are engaged to separate centrosomes biologically [92]. This suggests that the development of $\mathrm{Eg} 5$ inhibitors with an alternative mode of action, for example, ATP competitive, may present a potential avenue for investigation to remedy the low clinical efficacy.

\section{Resistance to kinesin inhibitors}

A major challenge in the area of cancer chemotherapy is the development of drug resistance. Known resistance mechanisms to kinesins can include upregulation of alternative pathways (as discussed in the 'Clinical outcomes with Eg5 inhibitors' section), expression of efflux pumps as seen with the CENP-E inhibitor GSK923295 [93] or mutations within the target protein. The emergence of point-mutations conferring resistance to Eg5 inhibitors targeting the L5/ $2 / \alpha 3$ site has already been reported [94-97], representing another challenge in the clinical application of $\mathrm{Eg} 5$ inhibitors.

\section{Resistance to chemical tools}

Studies have shown that mutations in the induced-fitbinding pocket of $\mathrm{Eg} 5$ can confer drug resistance to the inhibitors STLC and monastrol, both biochemically [96] and in a cellular context [97]. The introduction of several single-point mutations by alanine scanning mutagenesis led to resistance or partial resistance to monastrol and/or STLC, strongly suggesting that many different residues are equally important for drug binding. Three mutant strains (R119A, D130A and L214A; see Supplementary Figure 1) conferred significant resistance to both chemical probes in cell-based assays, characterized by the ability of cells to form normal bipolar spindles [97]. Structural explanations have been offered as to why these three particular residues appear to be important for effective inhibitor binding. Arg119 is located at the entrance of the binding pocket and forms part of the pocket to which elements of both monastrol (phenol ring) and STLC (trityl motif) make hydrophobic interactions. The side chains of Asp130 (loop 5) and Leu214 (helix $\alpha 3$ ) point toward both inhibitors, making favorable hydrophobic contacts or $\mathrm{CH}-\pi$ interactions (refer to the 'Monastrol \& analogs' and '( $S$ )-Trityl-L-cysteine (STLC) \& analogs' sections for Monastrol and STLCbinding modes, respectively). Curiously, these three drug-resistant strains can confer resistance to monastrol and STLC in the presence of wild-type Eg5, suggesting that an Eg5 tetramer consisting of a combination of drugsensitive and drug-resistant motor domains may still be able to generate force and motility [97]. The phenomenon of drug-sensitive proteins pairing with insensitive ones to give a functional oligomer is well documented in other contexts, for example, the transactivation of Raf kinase isoform heterodimers upon inhibitor binding resulting in paradoxical activation of the MAPK signaling pathway in cells expressing wild-type B-Raf [98,99]. Encouragingly, the development of 'paradox-breaking' pan-Raf inhibitors has been reported [100]. This suggests that if it

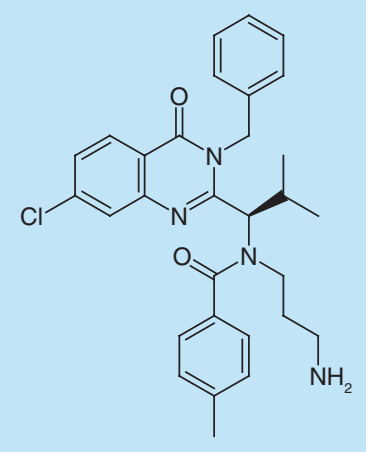

19

$\mathrm{K}<1 \mathrm{nM}$

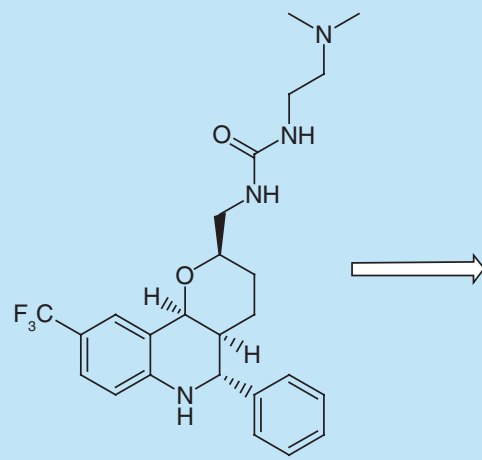

23

$\mathrm{IC}_{50} 8 \mathrm{nM}$

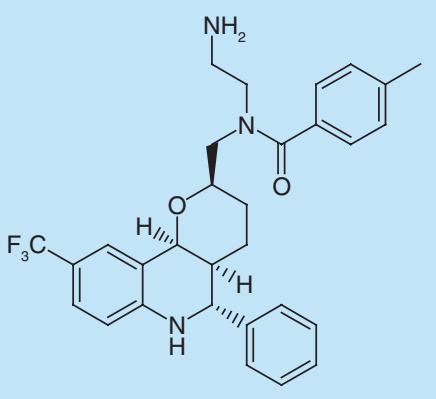

27

$\mathrm{IC}_{50} 65 \mathrm{nM}$

Figure 9. Scaffold-hopping approach toward an EMD-534085 (23)-ispinesib (19) hybrid compound 27 [66]. 
were possible to develop an inhibitor targeting multiple mutants of Eg5, this could overcome this mechanism of kinesin resistance.

$\mathrm{Eg} 5$ mutants resistant to clinical candidates have been identified, highlighting a significant risk of the emergence of resistance in patients treated with an $\mathrm{Eg} 5$ inhibitor. Long-term exposure of HCT116 colorectal cell lines to the clinical candidate ispinesib generated an ispinesibresistant cell line which was more than 3000 -fold less sensitive to the drug, but not cross-resistant to other cytotoxic drug including tubulin-targeting agents [101]. DNA sequencing of the $\mathrm{Eg} 5$ motor domain from the resistant cell line identified the two key point-mutations conferring resistance as D130V (also one of the pointmutation residues conferring resistance to monastrol and STLC) and A133D. Potency determinations for ispinesib against the two mutant strains of $\mathrm{Eg} 5$ revealed that the D130V is the more resistant of the two [102]. The ability of the mutations to confer resistance was rationalized as a result of their effect on the integrity of the binding pocket. D130 and A133 are involved in an extensive network of hydrogen-bond interactions, which has the effect of stabilizing the loop L5 in an inward conformation essential for inhibitor binding [102]. These same mutations have been found to also confer resistance to the second-generation Eg5 inhibitor SB-743921.

Further investigation of the resistance mechanism using calorimetry showed that mutated forms of $\mathrm{Eg} 5$ had improved enthalpic interactions with SB-743921, which is in contrast to the common assumption that mutations which result in drug resistance involve steric or electrostatic repulsion, leading to an enthalpically less favorable complex. The crystal structure of inhibitor-bound Eg5 A133D mutant did not reveal any obvious repulsive interactions compared with that of the wild-type protein [95].

The use of calorimetry in combination with modeling studies proposed that the resistance of $\mathrm{Eg} 5$ toward SB-743921 was through reduced flexibility of the protein as a result of local rearrangement of the hydrogenbonding interactions and salt bridging in the allosteric pocket. In the wild-type protein, Arg138, Ala133 and Asp130 form a network of hydrogen bonds and there is a salt bridge which exists between Glu128 and Lys 207 [95,103]. In the A133D variant, Arg138 formed a salt bridge with the newly introduced carboxylate of Asp133, breaking the bridge between Glu128 and Lys207 and allowing a new hydrogen-bond interaction to form between Lys207 and His141. These changes rigidify the overall complex. Reduced flexibility is typically associated with an entropy penalty which could account for some of the reduced affinity of the ligand toward Eg5. Additionally, key residues which are most efficient in energy exchange with their surroundings act as 'energy gates' [104], which communicate information via correlated residue fluctuations. In wild-type Eg5, residue A133 was identified as such an energy gate - able to transmit a perturbation through the protein to the nucleotide site when binding the inhibitor. When A133 was mutated, the energy gate was no longer present in the allosteric pocket, preventing transmission of allosteric inhibition. Crucially, energy gates within the nucleotide site are unaffected, enabling normal ATP hydrolysis and thus Eg5 motor function. This unpredictable phenomenon has been termed 'resistance by allostery' $[95,103]$. The key residues affected by point mutations in the $\mathrm{L} 5 / \alpha 2 / \alpha 3$-binding site are highlighted in Supplementary Figure 1. The authors of this study recommended that if a mutation arises which produces allosteric resistance, the focus should not be on identifying a new inhibitor of the same site, but identifying an alternative binding site with an alternative mode of inhibition to overcome the problem [95].

Since the development of inhibitors of HSET lags those of $\mathrm{Eg} 5$, and the binding mode of current inhibitors is unknown, it is not yet possible to determine whether HSET is susceptible to such 'resistance by allostery'. However, given the high degree of structural similarity between the kinesin families, similar phenomena may be likely to occur. Identifying where and how inhibitors bind and which residues are susceptible to inhibitor-induced mutations is likely to be important for the development of HSET inhibitors as well as for future $\mathrm{Eg} 5$ inhibitors.

The mutant strains of Eg5 may prove to be useful screening tools in the discovery of future $\mathrm{Eg} 5$ inhibitors. The STLC-resistant cell lines D130A and L214A have shown utility in distinguishing inhibitors, which bind to alternative pockets from the classical L5/ $22 / \alpha 3$-targeted inhibitors without the requirement of existing structural information. These cell lines were shown to be sensitive to a series of ATP-competitive inhibitors, which have been hypothesized to bind in a novel allosteric site [105].

\section{An alternative allosteric site}

The 'resistance by allostery' effect observed with SB-743921 led to the suggestion that an inhibitor targeting a novel site would resolve this [95]. Additionally, given the doubts regarding the effectiveness of $\mathrm{Eg} 5$ L5/ $/ 2 / \alpha 3$ allosteric inhibitors [92], the identification of novel inhibitors with an alternative mode of action is attractive. Intriguingly, a second allosteric site within Eg5 has been identified with chemical tools cocrystallized in the pocket between the $\alpha 4$ and $\alpha 6$ helices (Table 4).

\section{Biphenyl-based inhibitors}

In 2007, a set of biphenyl-based inhibitors of Eg5 was identified following a high-throughput screening 
campaign. Iterative medicinal chemistry to improve potency led to the identification of the highly potent inhibitors GSK-1 (28) and GSK-2 (29). Further characterization of these analogs revealed that they were firstin-class ATP-competitive inhibitors of Eg5 [106]. GSK-1 and GSK-2 bound to the ispinesib-resistant D130V and A133D mutants of Eg5, indicating that a binding site distinct to that of previous inhibitors was occupied. GSK-1 also showed potent activity in the HCT116 colon cancer cell line $\left(\mathrm{IC}_{50}=36 \mathrm{nM}\right)$, giving a phenotype indistinguishable from the classical monastrol phenotype despite the alternative mode of action.

A combination of biochemical and biophysical techniques was used to determine the inhibitor-binding site. Preparation of a moderately active photo-labile analog containing a reactive phenyltrifluoromethyldiazirine motif (GSK-3, 30) allowed covalent modification of the $\mathrm{Eg} 5$ protein. Labeled $\mathrm{Eg} 5$ motor domain was purified from the required microtubules, and subsequently digested with trypsin. Analysis by MALDI-TOF revealed residues $284-297$ as the site of ligand incorporation, and further interpretation of the data specifically identified Leu295 as the site of labeling. Leu295 is located in the middle of helix $\alpha 4$ with its side chain pointing toward the $\alpha 4 / 6$ interface, and molecular dynamics studies revealed that a small pocket between the switch II $\alpha 4 / 6$ helices can develop, indicating that despite being ATP competitive, the binding mode is distinct from the nucleotide-binding site [106].

A range of biphenyl-based inhibitors with similar mode of action was investigated independently, resulting in the identification of PVZB1194 (31), an ATP-competitive inhibitor of Eg5 [107]. PVZB1194 was eventually successfully cocrystallized with nucleotide-unbound Eg5 (Figure 10A; see Figure 10B for overlay with monastrolADP-bound Eg5), owing to the ability of this ligand to bind to $\mathrm{Eg} 5$ without the presence of microtubules. The phenyl ring of Tyr104 formed a stacking interaction with the 3-fluoro, 4-trifluoromethylphenyl ring, while Tyr352 stacked with the sulfonamide phenyl group, which made additional van der Waals interactions with Leu292 and Leu293. A network of hydrogen bonds existed among Tyr104, Glu345 and Ser269, which formed the wall of the narrow hydrophobic-binding pocket. Finally, the trifluoromethyl group made a series of van der Waals interactions with Tyr104, Ile332, Ala334, Tyr352 and Ala353 toward the bottom of the binding cleft. Interestingly, despite the high degree of potency for PVZB1194 for $\mathrm{Eg} 5$, there were no direct hydrogen-bonding interactions observed between ligand and protein [111].

The crystal structure showed that the neck linker was in a docked conformation, which appeared to stabilize the bottom of the PVZB1194-binding pocket. The importance of the neck-linker conformation for
PVZB1194 binding was investigated using a deletion mutant with the neck linker absent from the structure. While the ATPase function was retained, PVZB1194 was found to be 40-fold less potent against this truncated variant, confirming the requirement of a neck-linker-docked conformation for inhibitor binding. Consistent with the biochemical result that the biphenyl-based compounds were ATP competitive, the nucleotide-binding site was void of ATP or ADP. The surface model of the ATP-binding site showed that there was insufficient space to accommodate the nucleotide as a result of Glu129 and Thr107 occupying the site. Binding of PVZB1194 caused Tyr104 of strand $\beta 3$, which forms part of the bottom of the inhibitor pocket, to move through $1.8 \AA$ which in turn caused Thr107 to shift by $6.8 \AA$, altering the conformation of the ATP-binding site and preventing nucleotide binding.

The novel mode of action of biphenyl-based Eg5 inhibitors has stimulated further research. Improvements in the potency of the ATP-competitive class of inhibitors were achieved by introducing conformational restriction about the biphenyl junction, through development of carboline and carbazole scaffolds, resulting in reduced entropy loss (e.g., 32) [108]. Unfortunately, the planarity of the carbazole compounds resulted in very poor aqueous solubility, rendering the series unsuitable for further development. Ring opening to give a diarylamine-based scaffold gave equipotent ATP-competitive compounds with improved solubility (e.g., 33) [109].

\section{Benzimidazole-based inhibitors}

In 2010, the binding mode of a novel set of substituted benzimidazoles shown to target Eg5 was reported. The ability of these compounds to bind to a novel site was proposed after it was observed that they bound to $\mathrm{Eg} 5$ in the presence of ispinesib. Direct binding to D130V, A133D and the D130V-A133D double Eg5 mutants was also observed, further strengthening the hypothesis that a second independent binding site was targeted. The benzimidazole compounds were initially shown to be ATP noncompetitive, which is in contrast to the biphenyl second-site binders [110]. Confirmation of the binding site for the aminobenzimidazole-based compound BI8 (34) was accomplished with the aid of a crystal structure, published in 2013 [112].

Occupying a similar region as the biphenyl compounds, BI8 was found to bind in the pocket formed by $\alpha 4$ of the switch II cluster, $\alpha 6$ which precedes the neck-linker region and the $\beta 3$ strand with helices $\alpha 4$ and $\alpha 6$ shifting approximately $2 \AA$ to accommodate the inhibitor (Figure 11). The neck linker was undocked but the structure most resembled the intermediate inhibitor-bound state, as previously described for the classical L5/ $/ 2 / \alpha 3$ inhibitors. The potency 
Review Myers \& Collins

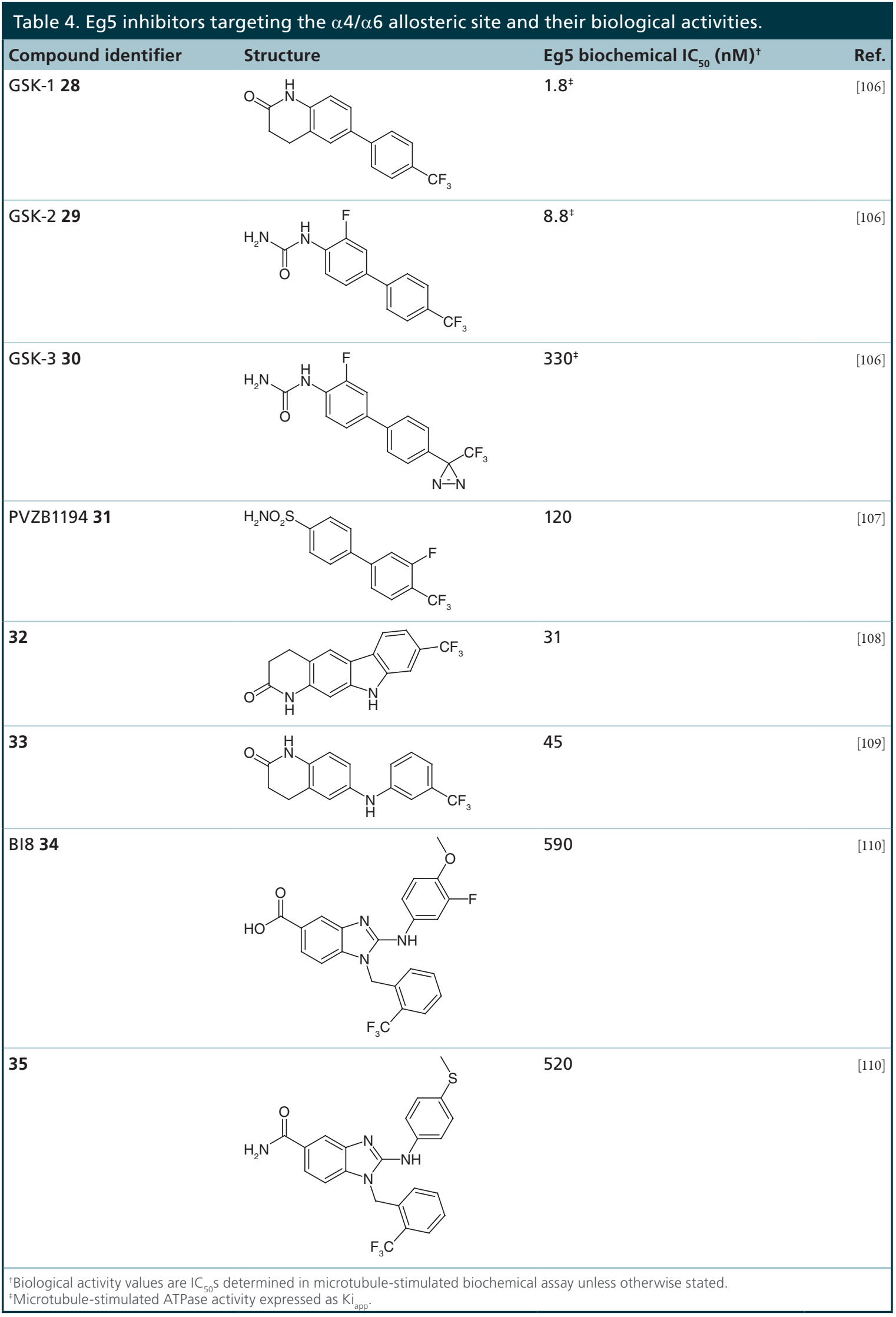

482

Future Med. Chem. (2016) 8(4)

future science group

fsh 
of BI8 for Eg5 could be explained as a result of an extensive network of aromatic interactions.

While there are some similarities between the binding of BI8 and PVZB1194 to Eg5, such as shared binding to residues Tyr104, Tyr352 and Leu292, there are some striking differences. Notably the neck linker is docked with PVZB1194 bound and undocked with BI8. The difference in the position of the neck linker in the inhibitor-bound structure also rendered BI8 different from L5/ $\alpha 2 / \alpha 3$ inhibitors such as STLC, whose crystal structure demonstrated that the final inhibitorbound state was with the neck linker in the docked conformation. Additionally, the $\beta 3$ strand which forms the bottom of the pocket was unaffected by BI8 binding, resulting in an ATP-noncompetitive mode of action [112].

Interestingly, toward the end of the crystal structure refinement for BI8-Eg5, electron density in a site which overlapped both the classical and newly identified allosteric pockets was detected. Parts of the inhibitor in this second site were found to be disordered, which suggested either a greater degree of flexibility and/or lower inhibitor occupancy. However, this raised concerns to whether the majority of potency observed in biochemical assays was due to binding at the $\alpha 2 / \alpha 3$ site, rather than the crystallographically identified novel a $4 / a 6$ site. Deconvolution of the site responsible for the measured inhibition was achieved using two parallel biophysical methods, isothermal titration calorimetry competition and surface plasmon resonance analysis, which confirmed the $\alpha 4 / \alpha 6$ site to be the single high-affinity site in this instance. Measurement of the strength of hydrogen-bonding interactions in the $\alpha 2 / \alpha 3$ site further supported that this was the weaker affinity binding site [112]. Counter screening of the benzimidazole-based Eg5 inhibitors against HSET highlighted a number with weaker HSET inhibitory activity [113].

\section{Future perspective}

The clinical data thus far for $\mathrm{Eg} 5$ inhibitors as a monotherapy have been largely disappointing. However, recently published clinical trial data with 4SC-205 showed that stable disease was achieved with the Eg5 inhibitor monotherapy dosed continuously, suggesting that refinement of the dosing regimen may be a viable strategy to overcome the low fraction of mitotic cells in slow-growing tumors [85]. Tackling this 'proliferation paradox' is likely to be important not only for mitotic kinesins but also for other agents acting predominantly in mitosis.

While some combinations of $\mathrm{Eg} 5$ inhibitors with other chemotherapies have also shown limited benefit in clinical trials, for example, SB-715992 in combination with docetaxel in solid tumors, preclinical studies have suggested that the kinesin inhibitors may be effective in combination with other agents, particularly an Aurora A [91] or KIF15/TPX2 inhibitor [89] should one reach the clinic. Such combinations may provide an opportunity to address functional redundancy among kinesins and improve the efficacy of Eg5 inhibitors. This highlights the
(A)

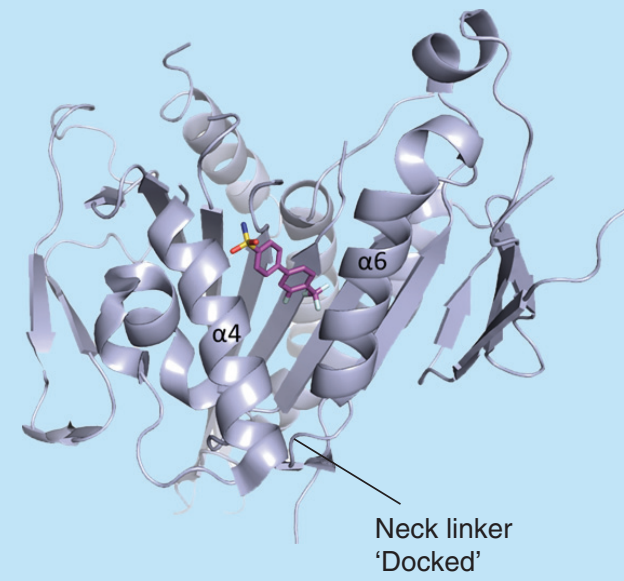

(B)

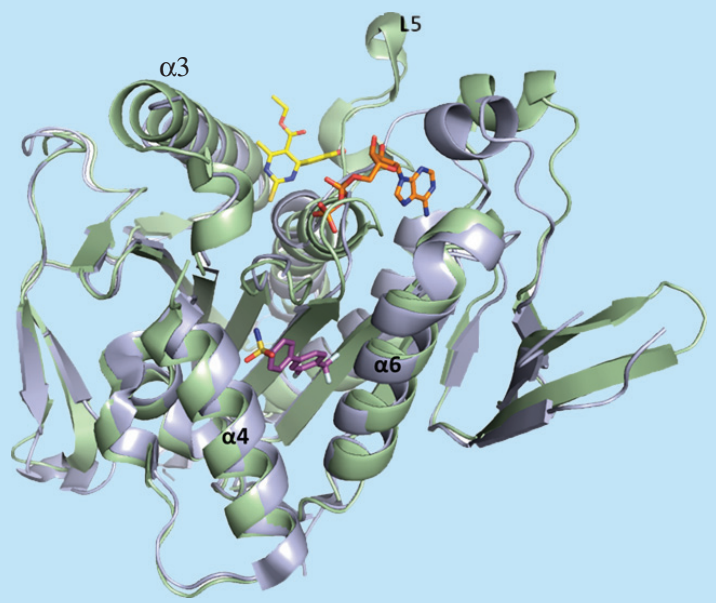

Figure 10. Binding of PVZB1194 to the second allosteric site in Eg. (A) Crystal structure of the ATP-competitive Eg5 inhibitor PVZB1194 (31, magenta), bound in the $\alpha 4 / \alpha 6$ allosteric site (PDB 3WPN). The neck linker is in a docked conformation. (B) Overlay of PVZB1194 (31, magenta), bound in the $\alpha 4 / \alpha 6$ allosteric site (light blue, PDB 3WPN) with the ATP noncompetitive inhibitor monastrol (1, yellow) bound in the L5/ $22 / \alpha 3$ allosteric site (pale green, PDB $1 \times 88$ ). Bound ADP is shown in stick form (orange). 


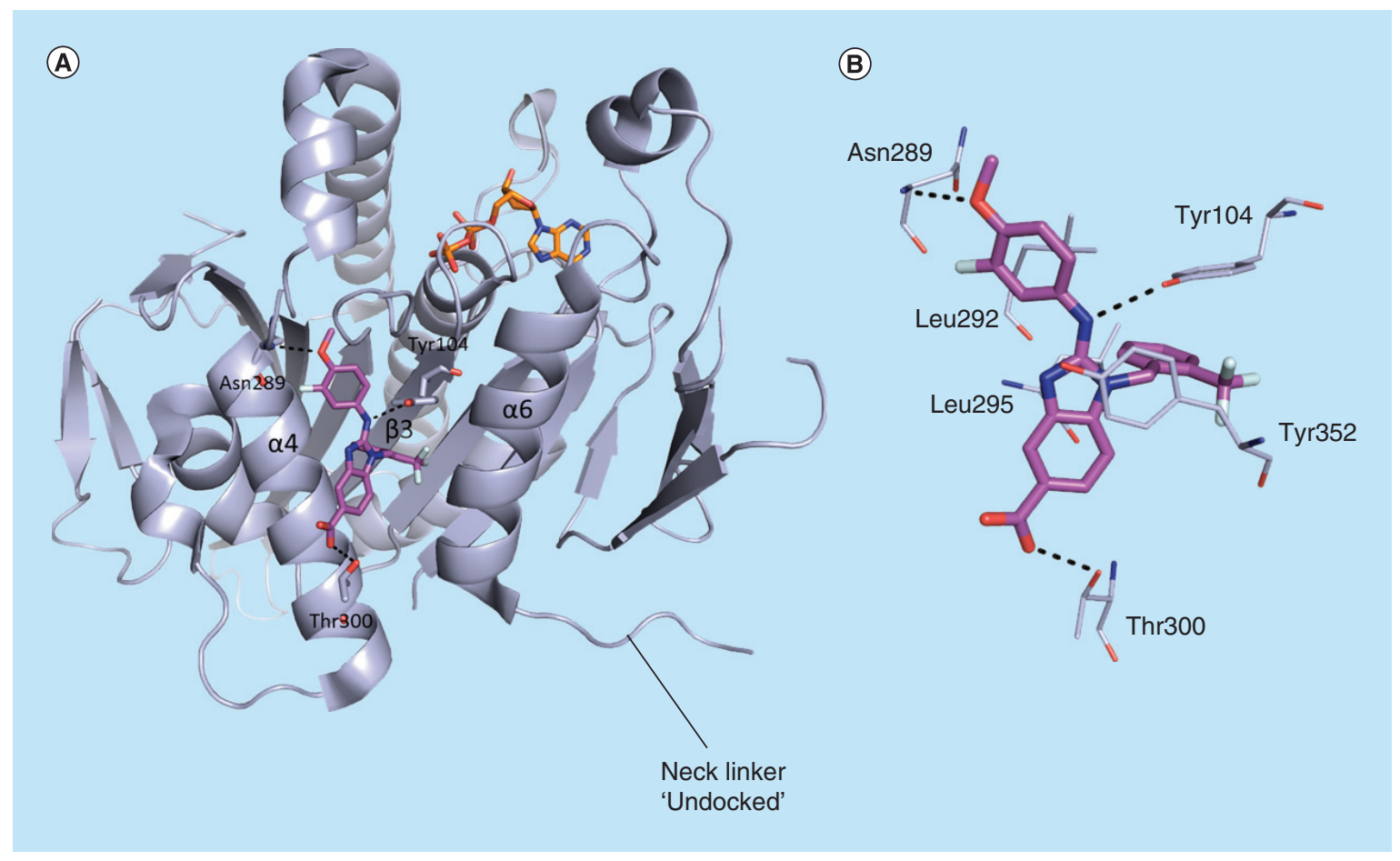

Figure 11. Binding of BI8 to the second allosteric site of Eg5. (A) Crystal structure of the ATP noncompetitive inhibitor BI8 (34, magenta) bound in the $\alpha 4 / \alpha 6$ allosteric site (PDB 3ZCW). Water molecules are not shown. The neck linker is in an undocked conformation and ADP (orange sticks) can be seen in the nucleotide-binding site. Key hydrogen bonds are shown (dotted lines). (B) Detailed ligand view of BI8 bound in Eg5. The phenol ring of Tyr352 forms a face-to-face stacking interaction with the benzimidazole ring and makes a watermediated interaction with a nitrogen atom of the imidazole ring (not shown). Similarly, Tyr $104 \pi$-stacks with the trifluoromethylbenzyl motif and a strong hydrogen bond between the secondary amino group of $\mathrm{BI} 8$ and the phenol of Tyr104 can be observed. The inhibitor's 3-fluoro-4-methoxyphenyl ring is in close proximity to the side chain of Leu292, and Leu295 is buried upon binding. The carboxylate group of BI8 makes a strong hydrogen bond with Thr300, strengthened with a second hydrogen bond to Arg297 via a water molecule (not shown).

requirement for preclinical selection of combination therapies based on mechanistic rationales, and parallels the clinical experience in oncology with targeted agents for signaling kinases. Here, functional redundancy between kinase signaling cascades is a frequent mode of resistance to monotherapy and may be tackled through drug combinations that simultaneously suppress the alternative pathways [114]. Should Eg 5 inhibitors with an alternative modality (e.g., ATP competitive) reach the clinic, combination of these inhibitors with the classical ATP noncompetitive inhibitors (such as ispinesib) may also be of interest. Dual inhibition of $\mathrm{Eg} 5$ at both allosteric-binding sites may result in increased clinical efficacy by fully arresting the motility of $\mathrm{Eg} 5$ along microtubules and may also reduce the chances of drug-resistant strains of Eg5 emerging by targeting two distinct sites on the same protein simultaneously.

As is the case with many chemotherapeutic strategies against cancer, the development of resistance to $\mathrm{Eg} 5$ inhibitors is highly likely, particularly those targeting the L5/ $\alpha 2 / \alpha 3$-binding site. The unpredictable 'resistance by allostery' effect observed following inhibition of $\mathrm{Eg} 5$ by SB-743921 led to the suggestion that inhibitors occupying an alternative allosteric site may be able to overcome this [95]. Thus, characterization of where and how inhibitors bind and which residues are susceptible to mutation to generate inhibitor resistance is likely to be important in the future development of kinesin inhibitors. The identification of a second allosteric ligand-binding site within the $\mathrm{Eg} 5$ protein, with chemical tools cocrystallized in the pocket between the $\alpha 4$ and $\alpha 6$ helices, represents a new avenue for the development of inhibitors. Agents targeting this $\alpha 4 / \alpha 6$ site may not only have improved resilience to the drug resistance mechanisms affecting the $L 5 / \alpha 2 / \alpha 3$ allosteric site but also by inhibiting $\mathrm{Eg} 5$ in an ATP-competitive manner offer a mode of action which could block, rather than slow, microtubule sliding and lead to a more potent effect in cancer cells [92].

Achieving selectivity for Eg 5 over HSET has not proven to be a challenge in inhibitor development 
at the classical L5/ $\alpha 2 / \alpha 3$ allosteric site, which can be explained by the differences in the $\mathrm{L} 5$ region of the two proteins. However, to date no crystallographic evidence of HSET binding is available, therefore the binding sites of the two specific HSET inhibitors AZ82 and CW069 have not yet been elucidated. Interestingly, AZ82 has been identified as an ATP-competitive inhibitor of HSET. As the only ATP-competitive inhibitors of Eg5 identified to date occupy an allosteric site distinct to that of the L5/ 22 / $\alpha 3$ site, and do not directly interact with the ATP site, it is possible that the ATP-competitive mode of action of AZ82 also results from structural changes arising from occupation of a novel allosteric site.

The large number of $\mathrm{Eg} 5$ clinical candidates spread over several chemical scaffolds and the recent identification of HSET chemical tools demonstrate the tractability of kinesins to high-throughput screening and structural biology approaches to find and develop inhibitors. This is despite the high degree of flexibility shown by the proteins and the multiprotein nature of their interactions with microtubules. The structural biology elucidation of the complex mechanochemistry of the $\mathrm{Eg} 5$ kinesin has proceeded in tandem with a better understanding of how allosteric inhibitors interfere with the motor and binding functions. The similarities and differences between the druggable sites on Eg5 and HSET are not yet fully understood and inhibitor-bound structural information is likely to be crucial for the development of future HSET and Eg5 clinical candidates, and indeed other mitotic kinesins of pharmacological interest.

\section{Supplementary data}

To view the supplementary data that accompany this paper please visit the journal website at: www.future-science.com/ doi/full/10.4155/fmc.16.5

\section{Financial \& competing interests disclosure}

This work was supported by The Institute of Cancer Research and Cancer Research UK [CUK] (Grant no. C309/A11566). The authors (SM Myers and I Collins) are employees of the Institute of Cancer Research, which has a commercial interest in inhibitors of kinesins. Please note that all authors who are, or have been, employed by The Institute of Cancer Research are subject to a 'Rewards to Inventors Scheme,' which may reward contributors to a program that is subsequently licensed. The authors have no other relevant affiliations or financial involvement with any organization or entity with a financial interest in or financial conflict with the subject matter or materials discussed in the manuscript apart from those disclosed.

No writing assistance was utilized in the production of this manuscript.

\section{Open access}

This article is distributed under the terms of the Creative Commons Attribution License 4.0 which permits any use, distribution, and reproduction in any medium, provided the original author(s) and the source are credited. To view a copy of the license, visit http://creativecommons.org/licenses/by/4.0/

\section{Executive summary}

- Eg5 and HSET interpolar mitotic kinesins have opposing motor functions in mitotic spindle assembly

- Both present attractive cancer cell selective targets

- Structure-based undertanding of allosteric inhibition underpins the discovery of kinesin inhibitors

- Current challenges in Eg5 inhibitor clinical development, including poor efficacy and resistance, may be addressed by changes to drug schedule or inhibitors targeting novel allosteric sites

\section{References}

Papers of special note have been highlighted as:

- of interest; $\bullet \bullet$ of considerable interest

1 Matson DR, Stukenberg PT. Spindle poisons and cell fate: a tale of two pathways. Mol. Interv. 11(2), 141-150 (2011).

2 Greenberger L, Sampath D. Resistance to taxanes. In: Cancer Drug Discovery and Development: Cancer Drug Resistance. Teicher B (Ed.). Humana Press, NJ, USA, 329-358 (2006).

3 Rowinksy E. The vinca alkaloids. In: Holland-Frei Cancer Medicine (6th Edition). Kufe DW, Pollock RE, Weichselbaum RR et al. (Eds). BC Decker, Hamilton, ON, Canada (2003).

4 Wojcik EJ, Buckley RS, Richard J, Liu L, Huckaba TM, Kim S. Kinesin-5: cross-bridging mechanism to targeted clinical therapy. Gene 531, 133-149 (2013).
5 Wordeman L. How kinesin motor proteins drive mitotic spindle function: lessons from molecular assays. Semin. Cell Dev. Biol. 21, 260-268 (2010).

6 McGrath MJ, Kuo IFW, Hayashi S, Takada S. Adenosine triphosphate hydrolysis mechanism in kinesin studied by combined quantum-mechanical/molecular-mechanical metadynamics simulations. J. Am. Chem. Soc. 135, 8908-8919 (2013).

7 Cross RA, McAinsh A. Prime movers: the mechanochemistry of mitotic kinesins. Nat. Rev. Mol. Cell Biol. 15, 257-271 (2014).

8 Rath O, Kozielski F. Kinesins and cancer. Nat. Rev. Cancer 12, 527-539 (2012).

- Comprehensive review of the role of mitotic kinesins in cancer and drug discovery for these targets. 
9 Winey M, Bloom K. Mitotic spindle form and function. Genetics 190, 1197-1224 (2012).

10 Slangy A, Lane HA, d'Hérin P, Harper M, Kress M, Niggt EA. Phosphorylation by p34cdc2 regulates spindle association of human $\mathrm{Eg} 5$, a kinesin-related motor essential for bipolar spindle formation in vivo. Cell 83, 1159-1169 (1995).

11 Tanenbaum ME, MacŮrek L, Janssen A, Geers EF, AlvarezFernández M, Medema RH. Kif15 cooperates with Eg5 to promote bipolar spindle assembly. Curr. Biol. 19, 1703-1711 (2009)

12 Vanneste D, Takagi M, Imamoto N, Vernos I. The role of Hklp2 in the stabilization and maintenance of spindle bipolarity. Curr. Biol. 19, 1712-1717 (2009).

13 Mountain V, Simerly C, Howard L, Ando A, Schatten G, Compton DA. The kinesin-related protein, HSET, opposes the activity of $\mathrm{Eg} 5$ and cross-links microtubules in the mammalian mitotic spindle. J. Cell Biol. 147, 351-366 (1999).

14 Zhu C, Zhao J, Bibikova M et al. Functional analysis of human microtubule-based motor proteins, the kinesins and dyneins, in mitosis/cytokinesis using RNA interference. Mol. Biol. Cell 16, 3187-3199 (2005).

15 Cai S, Weaver LN, Ems-McClung SC, Walczak CE. Kinesin-14 family proteins HSET/XCTK2 control spindle length by cross-linking and sliding microtubules. Mol. Biol. Cell 20, 1348-1359 (2009).

16 Cai S, Weaver LN, Ems-McClung SC, Walczak CE. Proper organization of microtubule minus ends is needed for midzone stability and cytokinesis. Curr. Biol. 20, 880-885 (2010).

17 Marx A, Hoenger A, Mandelkow E. Structures of kinesin motor proteins. Cell Motil. Cytoskel. 66, 958-966 (2009).

- Comprehensive description of the structure of kinesin motor proteins.

18 Chandrasekaran G, Tatrai P, Gergely F. Hitting the brakes: targeting microtubule motors in cancer. Br. J. Cancer 113, 693-698 (2015).

19 Scarabelli G, Grant BJ. Mapping the structural and dynamical features of kinesin motor domains. PLoS Comput. Biol. 9, e1003329 (2013).

20 Turner J, Anderson R, Guo J, Beraud C, Fletterick R, Sakowicz R. Crystal structure of the mitotic spindle kinesin $\mathrm{Eg} 5$ reveals a novel conformation of the neck-linker. J. Biol. Chem. 276, 25496-25502 (2001).

21 Harrington TD, Naber N, Larson AG, Cooke R, Rice SE, Pate E. Analysis of the interaction of the Eg5 Loop5 with the nucleotide site. J. Theor. Biol. 289, 107-115 (2011).

22 Rice S, Lin AW, Safer D et al. A structural change in the kinesin motor protein that drives motility. Nature 402, 778-784 (1999).

23 Sarli V, Giannis A. Targeting the kinesin spindle protein: basic principles and clinical implications. Clin. Cancer Res. 14, 7583-7587 (2008).

24 Ding ST, Xing ND, Lu JJ et al. Overexpression of Eg5 predicts unfavorable prognosis in non-muscle invasive bladder urothelial carcinoma. Int. J. Urol. 18, 432-438 (2011).

25 Sun DQ, Lu JJ, Ding KJ et al. The expression of Eg 5 predicts a poor outcome for patients with renal cell carcinoma. Med. Oncol. 30, 476 (2013).

26 Liu M, Wang X, Yang Y et al. Ectopic expression of the microtubule-dependent motor protein $\mathrm{Eg} 5$ promotes pancreatic tumourigenesis. J. Pathol. 221, 221-228 (2010).

27 Castillo A, Morse HC, Godfrey VL, Naeem R, Justice MJ. Overexpression of $\mathrm{Eg} 5$ causes genomic instability and tumor formation in mice. Cancer Res. 67, 10138-10147 (2007).

28 Koller E, Propp S, Zhang H et al. Use of a chemically modified antisense oligonucleotide library to identify and validate $\mathrm{Eg} 5$ (kinesin-like 1) as a target for antineoplastic drug development. Cancer Res. 66, 2059-2066 (2006).

29 Rello-Varona S, Vitale I, Kepp O et al. Preferential killing of tetraploid tumor cells by targeting the mitotic kinesin $\mathrm{Eg} 5$. Cell Cycle 8, 1030-1035 (2009).

30 Hayashi N, Koller E, Fazli L, Gleave ME. Effects of Eg5 knockdown on human prostate cancer xenograft growth and chemosensitivity. Prostate 68, 1283-1295 (2008).

31 Marra E, Palombo F, Ciliberto G, Aurisicchio L. Kinesin spindle protein siRNA slows tumor progression. J. Cell. Physiol. 228, 58-64 (2013).

32 Carter BZ, Mak DH, Shi Y et al. Regulation and targeting of $\mathrm{Eg} 5$, a mitotic motor protein in blast crisis CML: overcoming imatinib resistance. Cell Cycle 5, 2223-2229 (2006).

33 Saijo T, Ishii G, Ochiai A et al. Eg5 expression is closely correlated with the response of advanced non-small cell lung cancer to antimitotic agents combined with platinum chemotherapy. Lung Cancer 54, 217-225 (2006).

34 Kwon M, Godinho SA, Chandhok NS et al. Mechanisms to suppress multipolar divisions in cancer cells with extra centrosomes. Gene. Dev. 22, 2189-2203 (2008).

35 Ogden A, Rida PCG, Aneja R. Let's huddle to prevent a muddle: centrosome declustering as an attractive anticancer strategy. Cell Death Differ. 19, 1255-1267 (2012).

36 Kleylein-Sohn JP, Pollinger B, Ohmer M et al. Acentrosomal spindle organization renders cancer cells dependent on the kinesin HSET. J. Cell Science 125, 5391-5402 (2012).

37 Li Y, Lu W, Chen D et al. KIFC1 is a novel potential therapeutic target for breast cancer. Cancer Biol. Ther. 16(9), 1316-1322 (2015).

38 Pawar S, Donthamsetty S, Pannu V et al. KIFC1, a novel putative prognostic biomarker for ovarian adenocarcinomas: delineating protein interaction networks and signaling circuitries. J. Ovarian Res. 7, 53 (2014).

39 Pannu V, Rida PCG, Ogden A et al. HSET overexpression fuels tumor progression via centrosome clusteringindependent mechanisms in breast cancer patients. Oncotarget 6, 6076-6091 (2015).

40 Grinberg-Rashi H, Ofek E, Perelman M et al. The expression of three genes in primary non-small cell lung cancer is associated with metastatic spread to the brain. Clin. Cancer Res. 15, 1755-1761 (2009). 
41 El-Nassan HB. Advances in the discovery of kinesin spindle protein $(\mathrm{Eg} 5)$ inhibitors as antitumor agents. Eur. J. Med. Chem. 62, 614-631 (2013).

42 Gartner M, Sunder-Plassmann N, Seiler J et al. Development and biological evaluation of potent and specific inhibitors of mitotic kinesin Eg5. Chem. Bio. Chem. 6, 1173-1177 (2005).

43 Mayer TU, Kapoor TM, Haggarty SJ, King RW, Schreiber SL, Mitchison TJ. Small molecule inhibitor of mitotic spindle bipolarity identified in a phenotype-based screen. Science 286, 971-974 (1999).

44 Kaan HYK, Ulaganathan V, Rath O et al. Structural basis for inhibition of $\mathrm{Eg} 5$ by dihydropyrimidines: stereoselectivity of antimitotic inhibitors enastron, dimethylenastron and fluorastrol. J. Med. Chem. 53, 5676-5683 (2010).

45 Garcia-Saez I, DeBonis S, Lopez R et al. Structure of human $\mathrm{Eg} 5$ in complex with a new monastrol-based inhibitor bound in the $R$ configuration. J. Biol. Chem. 282, 9740-9747 (2007).

46 Barsanti PA, Wang W, Ni ZJ et al. The discovery of tetrahydro- $\beta$-carbolines as inhibitors of the kinesin Eg5. Bioorg. Med. Chem. Lett. 20, 157-160 (2010).

47 Skoufias DA, DeBonis S, Saoudi Y et al. S-trityl-L-cysteine is a reversible, tight binding inhibitor of the human kinesin $\mathrm{Eg} 5$ that specifically blocks mitotic progression. J. Biol. Chem. 281, 17559-17569 (2006).

48 Kaan HYK, Weiss J, Menger D et al. Structure-activity relationship and multidrug resistance study of new $S$ trityl-L-cysteine derivatives as inhibitors of Eg5. J. Med. Chem. 54, 1576-1586 (2011).

49 Good JAD, Wang F, Rath O et al. Optimized $S$-trityl-Lcysteine-based inhibitors of kinesin spindle protein with potent in vivo antitumor activity in lung cancer xenograft models. J. Med. Chem. 56, 1878-1893 (2013).

50 Ogo N, Ishikawa Y, Sawada J, Matsuno K, Hashimoto A, Asai A. Structure-guided design of novel $l$-cysteine derivatives as potent KSP inhibitors. ACS Med. Chem. Lett. 6, 1004-1009 (2015).

51 Pinkerton AB, Lee TT, Hoffman TZ et al. Synthesis and SAR of thiophene containing kinesin spindle protein (KSP) inhibitors. Bioorg. Med. Chem. Lett. 17, 3562-3569 (2007).

52 Cochran JC, Gatial JE, Kapoor TM, Gilbert SP. Monastrol inhibition of the mitotic kinesin Eg5. J. Biol. Chem. 280, 12658-12667 (2005).

53 Yan Y, Sardana V, Xu B et al. Inhibition of a mitotic motor protein: where, how, and conformational consequences. J. Mol. Biol. 335, 547-554 (2004).

54 Kaan HY, Ulaganathan V, Hackney DD, Kozielski F. An allosteric transition trapped in an intermediate state of a new kinesin-inhibitor complex. Biochem. J. 425, 55-60 (2010).

-. Crystal structures indicating the pathway of structural changes upon inhibitor binding to $\mathrm{Eg} 5$.

55 Ogo N, Oishi S, Matsuno K, Sawada J, Fujii N, Asai A. Synthesis and biological evaluation of L-cysteine derivatives as mitotic kinesin Eg5 inhibitors. Bioorg. Med. Chem. Lett. 17, 3921-3924 (2007).
56 DeBonis S, Skoufias DA, Indorato R-L et al. Structure-activity relationship of $S$-trityl-L-cysteine analogues as inhibitors of the human mitotic kinesin Eg5. J. Med. Chem. 51, 1115-1125 (2008).

57 Wang F, Good JAD, Rath O et al. Triphenylbutanamines: kinesin spindle protein inhibitors with in vivo antitumor activity. J. Med. Chem. 55, 1511-1525 (2012).

58 Maliga Z, Xing J, Cheung H, Juszczak LJ, Friedman JM, Rosenfeld SS. A pathway of structural changes produced by monastrol binding to Eg5. J. Biol. Chem. 281, 7977-7982 (2006).

59 DeBonis S, Simorre J-P, Crevel I et al. Interaction of the mitotic inhibitor monastrol with human kinesin Eg5. Biochemistry 42, 338-349 (2003).

Watts CA, Richards FM, Bender A et al. Design, synthesis, and biological evaluation of an allosteric inhibitor of HSET that targets cancer cells with supernumerary centrosomes. Chem. Biol. 20, 1399-1410 (2013).

- Discovery of the HSET inhibitor CW069 and modelling of its binding mode.

61 Bender A, Young DW, Jenkins JL et al. Chemogenomic data analysis: prediction of small-molecule targets and the advent of biological fingerprint. Comb. Chem. High Throughput Screen. 10, 719-731 (2007).

62 Klabunde T. Chemogenomic approaches to drug discovery: similar receptors bind similar ligands. $\mathrm{Br}$. J. Pharmacol. 152, 5-7 (2007)

63 Yang B, Lamb ML, Zhang T et al. Discovery of potent KIFC1 inhibitors using a method of integrated highthroughput synthesis and screening. J. Med. Chem. 57, 9958-9970 (2014).

64 Wu J, Mikule K, Wang W et al. Discovery and mechanistic study of a small molecule inhibitor for motor protein KIFC1. ACS Chem. Biol. 8, 2201-2208 (2013).

- Discovery and mechanistic study of the HSET inhibitor AZ82.

65 Wood KW, Lad L, Luo L et al. Antitumor activity of an allosteric inhibitor of centromere-associated protein-E. Proc. Natl Acad. Sci. USA 107, 5839-5844 (2010).

66 Schiemann K, Finsinger D, Zenke F et al. The discovery and optimization of hexahydro- $2 H$-pyrano[3,2-c] quinolines (HHPQs) as potent and selective inhibitors of the mitotic kinesin-5. Bioorg. Med. Chem. Lett. 20, 1491-1495 (2010).

67 Good JAD, Skoufias DA, Kozielski F. Elucidating the functionality of kinesins: an overview of small molecule inhibitors. Semin. Cell Dev. Biol. 22, 935-945 (2011).

68 Good JAD, Berretta G, Anthony NG, Mackay SP. The discovery and development of Eg5 inhibitors for the clinic. In: Kinesins and Cancer. Kozielski F (Ed.). Springer, Dordrecht, Holland (2015).

69 Hollebecque A, Deutsch E, Massard C et al. A Phase I, dose-escalation study of the Eg5-inhibitor EMD 534085 in patients with advanced solid tumors or lymphoma. Invest. New Drug 31, 1530-1538 (2013). 
70 Bergnes G, Ha E, Feng B et al. Mitotic kinesin-targeted antitumor agents: discovery, lead optimization and anti-tumor activity of a series of novel quinazolinones as inhibitors of kinesin spindle protein (KSP). Abstracts of Papers 223rd. National Meeting of the American Chemical Society, Orlando, FL, USA, 7-11 April 2002.

71 Jackson JR, Gilmartin A, Dhanak D et al. A second generation KSP inhibitor, SB-743921, is a highly potent and active therapeutic in preclinical models of cancer. Clin. Cancer Res. 12(Suppl. 19), B11 (2006).

72 Theoclitou ME, Aquila B, Block MH et al. Discovery of (+) - $N$-(3-aminopropyl) - $N$-[1-(5-benzyl-3-methyl-4oxo-[1,2] thiazolo [5,4-d] pyrimidin-6-yl)-2-methylpropyl]4-methylbenzamide (AZD4877), a kinesin spindle protein inhibitor and potential anticancer agent. J. Med. Chem. 54, 6734-6750 (2011).

73 Jeay S, Ali S, Chen C-R et al. Discovery of a novel Eg5 kinesin inhibitor, ARQ 621, with potent antitumor activity while sparing bone marrow-derived cells. Cancer Res. 68(Suppl. 9), 656 (2008).

74 Zhang B, Liu J-F, Xu Y, Ng S-C. Crystal structure of HsEg 5 in complex with clinical candidate CK0238273 provides insight into inhibitory mechanism, potency, and specificity. Biochem. Biophys. Res. Commun. 372, 565-570 (2008).

75 Talapatra SK, Schuttelkopf AW, Kozielski F. The structure of the ternary Eg5-ADP-ispinesib complex. Acta Crystallogr. D 68, 1311-1319 (2012).

76 Cox CD, Coleman PJ, Breslin MJ et al. Kinesin spindle protein (KSP) inhibitors. 9. Discovery of (2S)-4-(2,5difluorophenyl) - $N$-[(3R4S)-3-fluoro-1-methylpiperidin4-yl]-2-(hydroxymethyl)- $N$-methyl-2-phenyl-2,5-dihydro$1 H$-pyrrole-1-carboxamide (MK-0731) for the treatment of taxane-refractory cancer. J. Med. Chem. 51, 4239-4252 (2008).

77 Cox CD, Breslin MJ, Mariano BJ et al. Kinesin spindle protein (KSP) inhibitors. Part 1: the discovery of 3,5-diaryl-4,5dihydropyrazoles as potent and selective inhibitors of the mitotic kinesin KSP. Bioorg. Med. Chem. Lett. 15, 2041-2045 (2005).

78 Cox CD, Garbaccio RM. Discovery of allosteric inhibitors of kinesin spindle protein (KSP) for the treatment of taxanerefractory cancer: MK-0731 and analogs. Anticancer Agents Med. Chem. 10, 697-712 (2010).

79 LoRusso P, Goncalves P, Casetta L et al. First-in-human phase 1 study of filanesib (ARRY-520), a kinesin spindle protein inhibitor, in patients with advanced solid tumors. Invest. New Drug 33, 440-449 (2015).

80 Wakui H, Yamamoto N, Kitazono S et al. A Phase 1 and dose-finding study of LY2523355 (litronesib), an Eg5 inhibitor, in Japanese patients with advanced solid tumors. Cancer Chemother. Pharmacol. 74, 15-23 (2014).

81 Lonial S, Shah JJ, Zonder J et al. Prolonged survival and improved response rates with ARRY-520 in relapsed/ refractory multiple myeloma (RRMM) patients with low $\alpha-1$ acid glycoprotein (AAG) levels: results from a Phase 2 study. Blood 122, 285 (2013).

82 Komlodi-Pasztor E, Sackett D, Wilkerson J, Fojo T. Mitosis is not a key target of microtubule agents in patient tumors. Nat. Rev. Clin. Oncol. 8, 244-250 (2011).
83 Dybdal-Hargreaves NF, Risinger AL, Mooberry SL. Eribulin mesylate: mechanism of action of a unique microtubuletargeting agent. Clin. Cancer Res. 21, 2445-2452 (2015).

84 Komlodi-Pasztor E, Sackett DL, Fojo AT. Inhibitors targeting mitosis: tales of how great drugs against a promising target were brought down by a flawed rationale. Clin. Cancer Res. 18, 51-63 (2012).

85 Mross KB, Scharr D, Richly H et al. Overcoming the proliferation rate paradox: clinical evaluation of a continuous dosing scheme of the novel oral $\mathrm{Eg} 5$ inhibitor 4SC-205. J. Clin. Oncol. 33(Suppl.), Abstract 2528 (2015).

86 Kufer TA, Silljé HHW, Körner R, Gruss OJ, Meraldi P, Nigg EA. Human TPX2 is required for targeting Aurora-A kinase to the spindle. J. Cell Biol. 158, 617-623 (2002).

87 Chowdhury A, Chowdhury S, Tsai MY. A novel Aurora kinase A inhibitor MK-8745 predicts TPX2 as a therapeutic biomarker in non-Hodgkin lymphoma cell lines. Leukemia Lymphoma 53, 462-471 (2011).

88 Klejnot M, Falnikar A, Ulaganathan V, Cross RA, Baas PW, Kozielski F. The crystal structure and biochemical characterization of Kif15: a bifunctional molecular motor involved in bipolar spindle formation and neuronal development. Acta Crystallogr. D 70, 123-133 (2014).

89 Hancock WO. Mitotic kinesins: a reason to delve into kinesin-12. Curr. Biol. 24, R968-R970 (2014).

90 Komarova NL, Boland CR. Cancer: calculated treatment. Nature 499, 291-292 (2013).

91 Ma HT, Erdal S, Huang S, Poon RYC. Synergism between inhibitors of Aurora A and KIF11 overcomes KIF15dependent drug resistance. Mol. Oncol. 8, 1404-1418 (2014)

92 Waitzman JS, Larson AG, Cochran JC et al. The loop 5 element structurally and kinetically coordinates dimers of the human kinesin-5, Eg5. Biophys. J. 101, 2760-2769 (2011).

- Inhibitors of $\mathrm{Eg} 5$ that target L5 and the nucleotide pocket target different aspects of motor activity.

93 Tcherniuk SO, Oleinikov AV. Pgp efflux pump decreases the cytostatic effect of CENP-E inhibitor GSK923295. Cancer Lett. 361, 97-103 (2015).

94 Jackson JR, Auger KR, Gilmartin A et al. A resistance mechanism for the KSP inhibitor ispinesib implicates point mutations in the compound binding site. Proceedings of the AACNCI-EORTC Molecular Targets and Cancer Therapeutics Meeting. Presented at: AACR, Philadelphia, PA, USA, 14-18 November, 2005.

95 Talapatra SK, Anthony NG, Mackay SP, Kozielski F. Mitotic kinesin Eg5 overcomes inhibition to the Phase I/ II clinical candidate $\mathrm{SB} 743921$ by an allosteric resistance mechanism. J. Med. Chem. 56, 6317-6329 (2013).

- Elucidation of the molecular basis of resistance to the $\mathrm{Eg} 5$ inhibitor SB-743921, termed 'resistance by allostery'.

96 Brier S, Lemaire D, DeBonis S, Forest E, Kozielski F. Molecular dissection of the inhibitor binding pocket of mitotic kinesin $\mathrm{Eg} 5$ reveals mutants that confer resistance to antimitotic agents. J. Mol. Biol. 360, 360-376 (2006). 
97 Tcherniuk S, van Lis R, Kozielski F, Skoufias DA. Mutations in the human kinesin Eg5 that confer resistance to monastrol and $S$-trityl-L-cysteine in tumor derived cell lines. Biochem. Pharmacol. 79, 864-872 (2010).

98 Poulikakos PI, Zhang C, Bollag G, Shokat KM, Rosen N. RAF inhibitors transactivate RAF dimers and ERK signalling in cells with wild-type BRAF. Nature 464, 427-430 (2010).

99 Hatzivassiliou G, Song K, Yen I et al. RAF inhibitors prime wild-type RAF to activate the MAPK pathway and enhance growth. Nature 464, 431-435 (2010).

100 Girotti MR, Lopes F, Preece N et al. Paradox-breaking RAF inhibitors that also target SRC are effective in drugresistant BRAF mutant melanoma. Cancer Cell 27, 85-96 (2015).

101 Jackson JR, Auger KR, Gilmartin A et al. A resistance mechanism for the KSP inhibitor ispinesib implicates point mutations in the compound binding site. Proceedings of the AACNCI-EORTC Molecular Targets and Cancer Therapeutics Meeting. AACR, Philadelphia, PA, USA 14-18 November, 2005.

102 Sheth PR, Basso A, Duca JS et al. Thermodynamics of nucleotide and inhibitor binding to wild-type and ispinesib-resistant forms of human kinesin spindle protein. Biochemistry 48, 11045-11055 (2009).

103 Viswanath ANI, Pae AN. Resistance by allostery: a novel perspective for Eg5-targeted drug design. J. Med. Chem. 56, 6314-6316 (2013).

104 Haliloglu T, Gul A, Erman B. Predicting important residues and interaction pathways in proteins using Gaussian network model: binding and stability of HLA proteins. PLoS Comput. Biol. 6, e1000845 (2010).

105 Indorato R-L, DeBonis S, Kozielski F, Garcia-Saez I, Skoufias DA. STLC-resistant cell lines as tools to classify chemically divergent $\mathrm{Eg} 5$ targeting agents according to their mode of action and target specificity. Biochem. Pharmacol. 86, 1441-1451 (2013).
106 Luo L, Parrish CA, Nevins N et al. ATP-competitive inhibitors of the mitotic kinesin KSP that function via an allosteric mechanism. Nat. Chem. Biol. 3, 722-726 (2007).

107 Matsuno K, Sawada J, Sugimoto M, Ogo N, Asai A. $\mathrm{Bis}$ (hetero)aryl derivatives as unique kinesin spindle protein inhibitors. Bioorg. Med. Chem. Lett. 19, 1058-1061 (2009).

108 Takeuchi T, Oishi S, Watanabe T et al. Structure-activity relationships of carboline and carbazole derivatives as a novel class of ATP-competitive kinesin spindle protein inhibitors. J. Med. Chem. 54, 4839-4846 (2011).

109 Takeuchi T, Oishi S, Kaneda M et al. Kinesin spindle protein inhibitors with diaryl amine scaffolds: crystal packing analysis for improved aqueous solubility. ACS Med. Chem. Lett. 5, 566-571 (2014).

110 Sheth PR, Shipps GW, Seghezzi W et al. Novel benzimidazole inhibitors bind to a unique site in the kinesin spindle protein motor domain. Biochemistry 49, 8350-8358 (2010).

111 Yokoyama H, Sawada J, Katoh S et al. Structural basis of new allosteric inhibition in kinesin spindle protein $\mathrm{Eg} 5$. ACS Chem. Biol. 10, 1128-1136 (2015).

-. Crystal structure of PVZB1194 bound in the $\alpha 4 / \alpha 6 \mathrm{Eg} 5$ allosteric site.

112 Ulaganathan V, Talapatra SK, Rath O, Pannifer A, Hackney DD, Kozielski F. Structural insights into a unique inhibitor binding pocket in kinesin spindle protein. J. Am. Chem. Soc. 135, 2263-2272 (2013).

-. Crystal structure of BI8 bound in the $\alpha 4 / \alpha 6 \mathrm{Eg} 5$ allosteric site.

113 Lahue BR, Ma Y, Shipps Jr GW, Seghezzi W, Herbst R. Substituted benzimidazoles: a novel chemotype for small molecule hKSP inhibitors. Bioorg. Med. Chem. Lett. 19, 3405-3409 (2009).

114 Workman P, Al-Lazikani B, Clarke PA. Genome-based cancer therapeutics: targets, kinase drug resistance and future strategies for precision oncology. Curr. Opin. Pharmacol. 13, 486-496 (2013). 